\title{
Non-LTE line formation for Pr II and Pr III in A and Ap stars ${ }^{\star}$
}

\author{
L. Mashonkina ${ }^{1,2}$, T. Ryabchikova ${ }^{2,3}$, A. Ryabtsev $^{4}$, and R. Kildiyarova ${ }^{4}$ \\ 1 Institut für Astronomie und Astrophysik der Universität München, Scheinerstr. 1, 81679 München, Germany \\ e-mail: lyuda@usm.1mu.de \\ 2 Institute of Astronomy, Russian Academy of Sciences, Pyatnitskaya 48, 119017 Moscow, Russia \\ e-mail: lima@inasan.ru \\ 3 Institute for Astronomy, University of Vienna, Türkenschanzstrasse 17, 1180 Vienna, Austria \\ ${ }^{4}$ Institute of Spectroscopy, Russian Academy of Sciences, 142190 Troitsk, Moscow region, Russia
}

Received 26 May 2008 / Accepted 3 November 2008

ABSTRACT

\begin{abstract}
Aims. Non-local thermodynamical equilibrium (non-LTE) line formation for singly-ionized and doubly-ionized praseodymium is considered for a range of effective temperatures between $7250 \mathrm{~K}$ and $9500 \mathrm{~K}$. We evaluate the influence of departures from LTE on Pr abundance determinations and determine a distribution of the Pr abundance in the atmosphere of the roAp star HD 24712 from a non-LTE analysis of the Pr II and Pr III lines.

Methods. A comprehensive model atom for Pr II/III is presented based on the measured and the predicted energy levels, in total, 6708 levels of PrII and Pr III and the ground state of Pr IV. Calculations of the Pr II energy levels and oscillator strengths for the transitions in Pr II and Pr III are described.

Results. The dependence of non-LTE effects on the atmospheric parameters is discussed. At $T_{\text {eff }} \geq 8000 \mathrm{~K}$, departures from LTE lead to the overionization of Pr II and, therefore, to systematically depleted total absorption in the line and positive abundance corrections. At the lower temperatures, different lines of Pr II may be either weakened or amplified depending on the line strength. The non-LTE effects strengthen the Pr III lines and lead to negative abundance corrections. Non-LTE corrections grow with effective temperature for the Pr II lines, and, in contrast, they decline for the Pr III lines. The Pr II/III model atom is applied to determine the Pr abundance in the atmosphere of the roAp star HD 24712 from the lines of two ionization stages. In the chemically uniform atmosphere with $[\mathrm{Pr} / \mathrm{H}]=3$, the departures from LTE may explain only a small part (approximately 0.3 dex) of the difference between the LTE abundances derived from the Pr II and Pr III lines ( $\simeq 2$ dex). We find that the lines of both ionization stages are fitted for the vertical distribution of praseodymium, where the $\operatorname{Pr}$ enriched layer with $[\operatorname{Pr} / \mathrm{H}] \geq 4$ exists in the outer atmosphere at $\log \tau_{5000}<-4$. The departures from LTE for Pr II/III are strong in the stratified atmosphere and have the opposite sign for the Pr II and Pr III lines. The praseodymium stratification analysis of roAp stars has to be performed based on non-LTE line formation. Using the revised partition function of Pr II and experimental transition probabilities, we determine the solar non-LTE abundance to be $\log (\operatorname{Pr} / \mathrm{H})_{\odot}=-11.15 \pm 0.08$.
\end{abstract}

Key words. atomic data - atomic processes - line: formation - stars: atmospheres - stars: chemically peculiar stars: individual: HD 24712

\section{Introduction}

Classical LTE analysis finds a strong disparity between the second and the first ions of the rare-earth elements (REE) in rapidly oscillating chemically peculiar (roAp) stars (Cowley \& Bord 1998; Cowley et al. 2000; Gelbmann et al. 2000; Ryabchikova et al. 2001; Kochukhov 2003; Ryabchikova et al. 2004). For a sample of 26 stars, Ryabchikova et al. (2004) highlighted a discrepancy between the abundances derived from the lines of singly ionized and doubly ionized atoms of neodymium and praseodymium, which typically exceeded 1.5 dex in roAp stars, but was substantially smaller, if present, in non-pulsating Ap stars (see also Kato 2003 and Ryabchikova et al. 2006, for hotter Ap stars HD 170973 and HD 144897). This excludes errors in the oscillator strengths as a possible reason for the abundance difference observed in roAp stars.

In our previous paper (Mashonkina et al. 2005, hereafter, referred to as Paper I), we investigated the Nd II and Nd III spectra of two roAp stars, $\gamma$ Equ and HD 24712, based on nonlocal thermodynamic equilibrium (non-LTE) line formation, and

* Based on observations collected at the European Southern Observatory, Chile, 274.D-5011. demonstrated that non-LTE effects could explain only 0.5 dex of the difference between the LTE abundances derived from the Nd II and Nd III lines and not the 1.5-2.0 dex observed for these stars. Mashonkina et al. (2005) returned, therefore, to the assumption of Ryabchikova et al. (2002) that the Nd anomaly observed in $\gamma$ Equ is caused by a stratified Nd distribution with the accumulation of the element in the uppermost atmospheric layers, above $\log \tau_{5000}=-8$ according to the LTE analysis. It was found that the effects of non-LTE on the Nd II and Nd III lines are strong in the stratified atmosphere, and they produce significant shifting of the Nd enriched layer with respect to the location determined in the LTE analysis. The required Nd overabundance in the layer is $[\mathrm{Nd} / \mathrm{H}]=4$ at $\log \tau_{5000}<-3.5$ for $\gamma \mathrm{Equ}$, and $[\mathrm{Nd} / \mathrm{H}]=4.5$ at $\log \tau_{5000}<-4.5$ for HD 24712 .

The present paper continues to investigate the rare-earth elements in stellar atmospheres based on the non-LTE line formation and is devoted to praseodymium. We study the statistical equilibrium (SE) of singly ionized and doubly ionized praseodymium, Pr II and Pr III, for a range of effective temperatures between $7250 \mathrm{~K}$ and $9500 \mathrm{~K}$, evaluate the influence of departures from LTE on Pr abundance determinations in the Sun, A and Ap type stars, consider the effects of non-LTE on Pr II/III 
in the atmosphere with non-uniform vertical distribution of $\mathrm{Pr}$, and finally determine empirically a stratification of $\mathrm{Pr}$ in the atmosphere of the roAp star HD 24712 from the non-LTE analysis of the Pr II and Pr III lines.

The paper is organized as follows. In Sect. 2, an extensive model atom for Pr II/III is introduced and theoretical calculations of the Pr II atomic structure and transition probabilities for Pr II and Pr III are presented. The programs and atmospheric models used in the line-formation calculations are described in Sect. 3. Section 4 investigates the departures from LTE for Pr II/III in the model atmospheres with homogeneous and stratified distribution of Pr. The non-LTE abundance corrections for the selected lines of Pr III and Pr II are given, depending on effective temperature. The solar Pr abundance is revised in Sect. 5, based on the improved partition function of Pr II. In Sect. 6, we determine the Pr abundance distribution in the atmosphere of the roAp star HD 24712 from the non-LTE analysis of the Pr II and Pr III lines, and discuss the influence of the uncertainties in atomic parameters on non-LTE modelling and our final results. Our conclusions and recommendations are given in Sect. 7.

\section{Model atom of PrII-Pr III}

A model atom provides the necessary atomic input data for specifying the SE equations and the opacities/emissivities for radiative-transfer calculations.

\subsection{Energy levels}

The lower levels in singly ionized Pr belong to the $4 \mathrm{f}^{3} 6 \mathrm{~s}$ configuration with the $\left({ }^{4} \mathrm{I}\right)^{5} \mathrm{I}$ ground term. Laboratory measurements (Ginibre 1989a,b; Furman et al. 2001; Ivarsson et al. 2001) provide 330 energy levels of PrII with an excitation energy $E_{\text {exc }} \leq 5.3 \mathrm{eV}$. Most known levels belong to singlet, triplet, and quintet terms of the $4 \mathrm{f}^{3} n l(n l=6 \mathrm{~s}, 5 \mathrm{~d}, 6 \mathrm{p})$ and $4 \mathrm{f}^{2} 5 \mathrm{~d} n l(n l=$ $5 \mathrm{~d}, 6 \mathrm{~s}, 6 \mathrm{p}$ ) electronic configurations. For some of the known energy levels, only the total angular momentum and assignment to an electronic configuration were given. The highest known levels of Pr II are separated by more than $5 \mathrm{eV}$ from the ground state of Pr III. The calculations with the Cowan (1981) code show that even below $5.3 \mathrm{eV}$ there are many unidentified energy levels. Due to this incomplete term system, we are unable to achieve a realistic statistical equilibrium of the atom.

For the present study, we calculate the energy levels of Pr II using the Cowan code (Cowan 1981). The measured levels of the odd $4 \mathrm{f}^{3} 6 \mathrm{~s}+4 \mathrm{f}^{3} 5 \mathrm{~d}+4 \mathrm{f}^{2} 5 \mathrm{~d} 6 \mathrm{p}$ configurations were fitted by taking into account the interactions with the $4 \mathrm{f}^{2} 6 \mathrm{~s} 6 \mathrm{p}, 4 \mathrm{f} 5 \mathrm{~d}^{3}, 4 \mathrm{f} 5 \mathrm{~d}^{2} 6 \mathrm{~s}$, and $4 \mathrm{f} 5 \mathrm{~d} 6 \mathrm{~s}^{2}$ configurations. The $4 \mathrm{f}^{2} 5 \mathrm{~d}^{2}, 4 \mathrm{f}^{2} 5 \mathrm{~d} 6 \mathrm{~s}, 4 \mathrm{f}^{2} 6 \mathrm{~s}^{2}, 4 \mathrm{f}^{3} 6 \mathrm{p}$, $4 \mathrm{f}^{4}, 4 \mathrm{f}^{2} 6 \mathrm{p}^{2}$, and $4 \mathrm{f} 5 \mathrm{~d}^{2} 6 \mathrm{p}$ configurations are included in the fitted matrix of the even system. The fitting has a standard deviation of $86 \mathrm{~cm}^{-1}$ for 129 odd and $72 \mathrm{~cm}^{-1}$ for 201 even levels. Highly excited levels were predicted from calculations of the energy structures of the even $4 f^{3}(7 p-9 p)$ and $4 f^{3}(5 f-6 f)$ configurations and the odd $4 f^{3}(7 s-9 s), 4 f^{3}(6 d-8 d), 4 f^{3} 5 g$, and $4 f^{2} 5 d 5 f$ configurations. In all unknown configurations, the average energies were taken according to Brewer (1971) predictions and the energy parameters of the $4 \mathrm{f}^{2}$ and $4 \mathrm{f}^{3}$ cores were scaled similarly to the known configurations, the other parameters being scaled by a factor 0.75 with respect to the corresponding Hartree - Fock values. As a result, an almost complete set of the levels below $70000 \mathrm{~cm}^{-1}(8.7 \mathrm{eV})$ was obtained consisting of 3938 energies. All known and predicted levels with $E_{\text {exc }} \leq 10.04 \mathrm{eV}$, in total 6539 levels of Pr II, were used to construct the model atom. They
Table 1. Partition functions for Pr II and Pr III.

\begin{tabular}{|c|c|c|c|}
\hline \multirow{2}{*}{$\begin{array}{l}\text { Temperature } \\
\text { (K) }\end{array}$} & \multicolumn{2}{|c|}{ Pr II } & \multirow{2}{*}{$\begin{array}{c}\text { Pr III } \\
\text { this study }\end{array}$} \\
\hline & Ginibre (1989) & this study & \\
\hline 3000 & 55 & 58 & 22 \\
\hline 4000 & 91 & 101 & 28 \\
\hline 5000 & 138 & 166 & 34 \\
\hline 6000 & 192 & 251 & 43 \\
\hline 7000 & 252 & 356 & 54 \\
\hline 8000 & 315 & 478 & 66 \\
\hline 9000 & 380 & 617 & 81 \\
\hline 10000 & 446 & 770 & 97 \\
\hline 11000 & 511 & 937 & 114 \\
\hline 12000 & 576 & 1117 & 132 \\
\hline 13000 & 639 & 1309 & 151 \\
\hline 14000 & 701 & 1514 & 170 \\
\hline 15000 & 762 & 1732 & 190 \\
\hline 16000 & 820 & 1962 & 211 \\
\hline 17000 & 878 & 2205 & 231 \\
\hline 18000 & 933 & 2460 & 252 \\
\hline 19000 & 986 & 2727 & 273 \\
\hline 20000 & 1038 & 3007 & 293 \\
\hline
\end{tabular}

are shown in Figs. 1 and 2 for the even and odd levels, respectively. The calculated high excitation levels provide the close collisional coupling of Pr II to the continuum electron reservoir.

We note that the calculations give a significantly higher partition function of PrII than laboratory levels only. This is illustrated in Table 1, where the partition function of Pr II is calculated by its definition for a temperature range $3000 \mathrm{~K}-20000 \mathrm{~K}$ using the measured energy levels from Ginibre (1989a,b) and the levels predicted by this study. In Sect. 5, we calculate the effect of the revised partition function on the Pr abundance determined from the solar Pr II lines.

For Pr III, the laboratory measurements provided 593 energy levels (Martin et al. 1978; Palmeri et al. 2000) of excitation energy up to $17.5 \mathrm{eV}$. In the range of stellar parameters in which we are concerned, there is no need to include the highly excited levels of Pr III in the model atom. They play a minor role in the population and depopulation of Pr III, because the next ionization stage Pr IV represents a negligible fraction of the Pr abundance. We, therefore, use the levels of the odd $4 f^{3}$ and the even $4 f^{2} 5 d$ and $4 \mathrm{f}^{2} 6 \mathrm{~s}$ electronic configurations with $E_{\text {exc }} \leq 6.7 \mathrm{eV}$. The term structure is shown in Fig. 3. The contribution of the Pr III energy levels omitted in the final model atom to the Pr III partition function is less than $0.01 \%$ at $T_{\mathrm{e}}=7250 \mathrm{~K}$.

Levels of the same parity with small energy differences were combined into a single level. The final model atom included 294 combined levels of Pr II, 54 combined levels of Pr III, and the ground state of PrIV.

\subsection{Radiative data}

The 15788 and 392 radiative bound-bound transitions in Pr II and PrIII, respectively, are included in SE calculations. Oscillator strengths $f_{i j}$ based on laboratory measurements of Lage \& Whaling (1976) or estimated from the observed line intensities of Meggers et al. (1976) are available only for 448 transitions in Pr II. They originate in the Kurucz \& Bell (1995) linelist and are accessible via the Vienna Atomic Line Data Base (VALD, Kupka et al. 1999). 


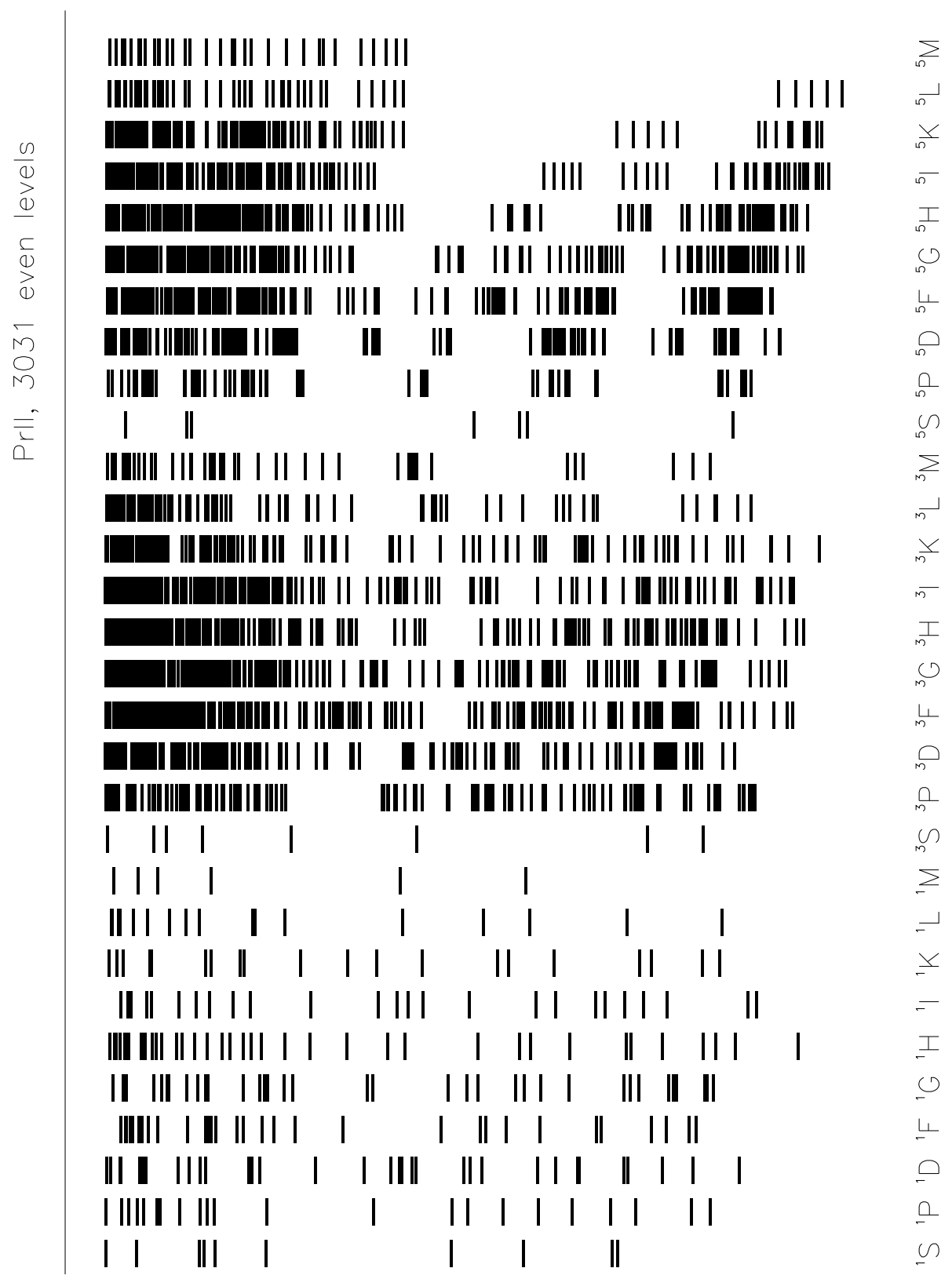

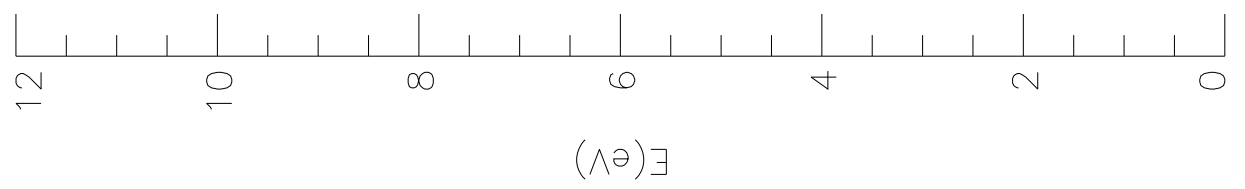

Fig. 1. The Pr II even term structure. 


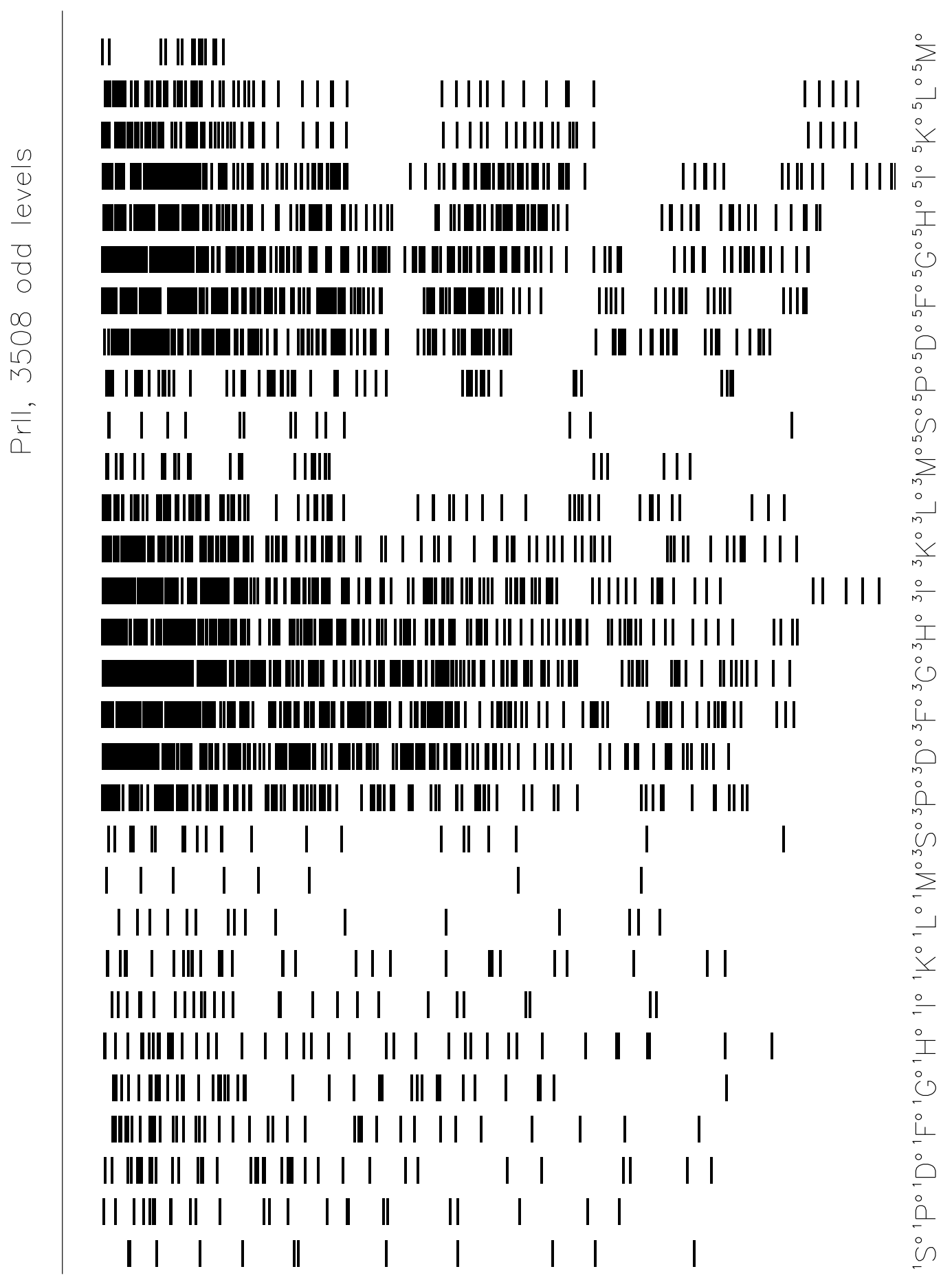

Fig. 2. The Pr II odd term structure.

For the majority of transitions in Pr II and all transitions in Pr III, we rely, therefore, on oscillator strengths computed in the present study. Calculations for Pr II are based on the wave functions obtained in the fitting of the known energy levels. All the Hartree-Fock transition integrals are scaled by a factor of 0.85 . The calculated lifetimes were compared with the accurate measurements of Scholl et al. (2002) and Biémont et al. (2003). Good agreement is found for levels that have the most significant contribution from the $4 \mathrm{f}^{3} 6 \mathrm{p}$ configuration: the ratio of the measured to calculated lifetime equals 1.1 with a standard deviation of 0.3 . For the levels with highly mixed wave functions, the corresponding quantity appears to be 0.7 with a standard deviation of 0.5 . Details of these calculations can be found on the ISAN webpage ${ }^{1}$ and will be presented in a forthcoming paper.

For Pr III, our approach follows that of Palmeri et al. (2000). Fitting of the energy levels is performed by accounting for the interactions in the odd $4 \mathrm{f}^{3}+4 \mathrm{f}^{2} 6 \mathrm{p}+4 \mathrm{f} 5 \mathrm{~d}^{2}+4 \mathrm{f} 5 \mathrm{~d} 6 \mathrm{~s}+4 \mathrm{f}^{2} \mathrm{~s}^{2}+4 \mathrm{f}^{2} 5 \mathrm{f}+$ $5 p^{5} 4 f^{4}$ and the even $4 f^{2} 5 d+4 f^{2} 6 d+4 f^{2} 6 s+5 p^{5} 4 f^{3} 5 d+5 p^{5} 4 f^{3} 6 s+$ $4 f 5 d 6 p+4 f 6 s 6 p+4 f 5 d 5 f$ complexes. Ab initio transition integrals are taken. For 7 levels with more than a $25 \%$ contribution

1 http://das101.isan.troitsk.ru/files/SPECTRA/PrII 


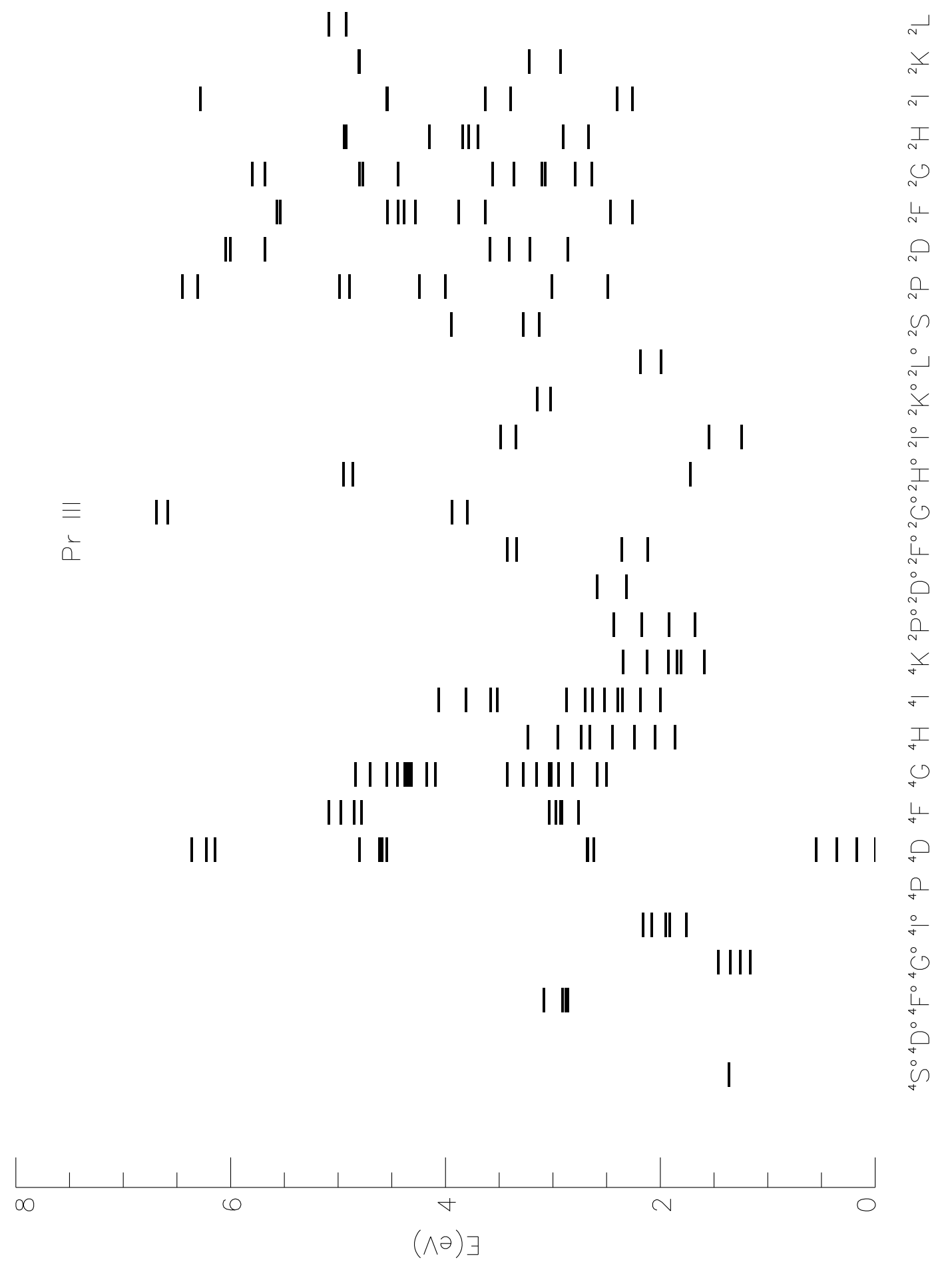

Fig. 3. The Pr III model atom.

from the $4 \mathrm{f}^{2} 6 \mathrm{p}$ configuration, the average ratio of the measured (Biémont et al. 2001) to calculated lifetimes is $1.17 \pm 0.25$. An exception is the $J=9 / 2$ level at $62535.6 \mathrm{~cm}^{-1}$, whose most significant contribution is from the $4 \mathrm{f} 5 \mathrm{~d}^{2}$ configuration and admixture of the $4 \mathrm{f}^{2} 6 \mathrm{p}$ configuration is about $11 \%$. For this level, the lifetime ratio appears to be 3.1. Details of the Pr III calculations relevant to this article can be found on the ISAN webpage ${ }^{2}$.

The photoionization cross-sections $\sigma_{\mathrm{ph}}$ are computed using the hydrogen approximation because no accurate data is available for the Pr II and Pr III levels. We assume that the

${ }^{2}$ http://das101.isan.troitsk.ru/files/SPECTRA/PrIII photoionization from any Pr II level ends in the ground state of $\operatorname{Pr}$ III, $4 \mathrm{f}^{3}{ }^{3} \mathrm{I}_{9 / 2}^{\circ}$. To take into account photoionization to the remaining levels of the $4 \mathrm{f}^{34} \mathrm{I}^{\circ}$ term, we multiply $\sigma_{\mathrm{ph}}$ by the ratio $g\left(4 \mathrm{f}^{3}{ }^{4} \mathrm{I}^{\circ}\right) / g\left(4 \mathrm{f}^{3}{ }^{4} \mathrm{I}_{9 / 2}^{\circ}\right) \simeq 5$. The photoionization from the $4 \mathrm{f}^{2} 5 \mathrm{~d} n l$ levels ends in the excited Pr III $4 \mathrm{f}^{2} 5 \mathrm{~d}$ levels. This is neglected for the following reason. Replacing the true ending state with the Pr III ground state ensures that each $4 \mathrm{f}^{2} 5 \mathrm{~d} n l$ level can be ionized more easy. Its ionization energy is reduced by more than $2 \mathrm{eV}$, and the photoionization rate is overestimated, in particular, for levels with a threshold in the ultraviolet (UV). At the same time, the photoionization rates of the $4 \mathrm{f}^{2} 5 \mathrm{~d} n l$ levels 
are underestimated due to ignoring multiple channels for their ionization. We cannot estimate the net effect due to absence of accurate atomic data.

\subsection{Collisional data}

The calculations of electron-impact excitation and ionization rates rely on theoretical approximations. We use the formula of van Regemorter (1962) for the allowed transitions and assume that the effective collision strength $\Upsilon=1$ for the forbidden transitions. Electron-impact ionization cross-sections are computed according to Drawin (1961).

The effects of the uncertainties in the adopted photoionization cross-sections and collisional rates on the final results are described in Sect. 6.3.

\section{Programs and model atmospheres}

The radiative transfer and statistical equilibrium equations are solved with a revised version of the DETAIL program (Butler \& Giddings 1985) using the accelerated lambda iteration following the extremely efficient method described by Rybicki \& Hummer (1991, 1992). Background opacities include the important bound-free and free-free transitions of hydrogen, helium, and the most abundant metals, and the effects of Rayleigh scattering, Thomson scattering, hydrogen lines, quasi-molecular Lyman $\alpha$ satellites due to $\mathrm{H}-\mathrm{H}$ and $\mathrm{H}-\mathrm{H}^{+}$collisions (Allard et al. 1998), and line opacity calculated with the line lists by Kurucz \& Bell (1995). The background opacities are sampled on a random grid of 4500 frequencies, to which the frequencies of the line profiles are added. The final non-LTE line-formation program samples roughly 150000 wavelengths between $500 \AA$ and $80000 \AA$.

The obtained non-LTE and LTE level populations are used to compute the emergent flux, line profiles, and equivalent widths with the code LINEC. The investigated lines and transitions of Pr II and Pr III are listed in Table 2. The wavelengths of Pr II lines are taken from Ginibre (1990). Ritz wavelengths are used for the Pr III lines.

Hyperfine-structure (HFS) affecting the Pr II lines is calculated explicitly with the HFS constants given by Ginibre (1989a), if available. No HFS data exist in the literature for the Pr III lines. Ivarsson et al. (2001, ILW) could not resolve HFS in any of the accurately measured Pr III lines, which means that HFS is not significant, although some lines exhibit asymmetric profiles of half-widths that exceed the half-width of the resolved HFS components in Pr II. The three of eight common Pr III lines are practically unaffected by HFS according to the ILW laboratory analysis. Half-widths of the other three lines are three to four times larger than those of the HFS unaffected lines, and they are comparable with the thermal half-widths of the Pr III lines in the atmosphere of HD 24712. The thermal half-width of the Pr lines corresponds to approximately $1 \mathrm{~km} \mathrm{~s}^{-1}$. Our simulation of HFS affecting the Pr III 5998 and 6053 lines with the strongest HFS broadening, according to the measurements of Ivarsson et al. (2001), leads to an increase in the theoretical equivalent widths, of $16 \mathrm{~m} \AA$ and $17 \mathrm{~m} \AA$, respectively, which transforms to a -0.20 dex and -0.24 dex decrease in the element abundance derived from these lines. In further analysis, the effects of HFS are not accounted for when modelling/analyzing the Pr III lines. In Sect. 6.2, we show that the two aforementioned lines provide results that are consistent with those for the Pr III lines with weak HFS broadening.
Table 2. Investigated transitions in Pr II and Pr III.

\begin{tabular}{|c|c|c|c|}
\hline$\lambda[\AA]$ & $E_{\mathrm{exc}}(\mathrm{eV})$ & \multicolumn{2}{|l|}{ Transition } \\
\hline Pr II & & & \\
\hline $4222.93^{1 *}$ & 0.05 & $4 f^{3} 5 d^{5} I_{5}^{\circ}-4 f^{3} 6 p^{5} K_{6}$ & $2-118^{2}$ \\
\hline $4449.83^{1 *}$ & 0.20 & $4 f^{3} 5 d^{5} I_{6}^{\circ}-4 f^{3} 6 p^{5} K_{6}^{0}$ & $3-118$ \\
\hline 5002.44 & 0.80 & $4 f^{3} 5 d^{5} K_{7}^{\circ}-4 f^{3} 6 p^{3}{ }^{6}{ }_{6}$ & $12-129$ \\
\hline $5110.76^{*}$ & 1.15 & $4 f^{3} 5 d^{5} L_{10}^{0}-4 f^{3} 6 p^{5} K_{9}^{6}$ & $26-143$ \\
\hline $5129.54^{*}$ & 0.65 & $4 f^{3} 5 d^{5} K_{6}^{\circ}-4 f^{3} 6 p^{5} I_{5}$ & $10-121$ \\
\hline $5135.14^{*}$ & 0.95 & $4 f^{3} 5 d^{5} K_{8}^{0}-4 f^{3} 6 p^{5} K_{8}$ & $16-134$ \\
\hline $5259.73^{1 *}$ & 0.63 & $4 f^{3} 5 d^{5} L_{7}^{0}-4 f^{3} 6 p^{5} K_{6}^{8}$ & $9-118$ \\
\hline $5292.62^{*}$ & 0.65 & $4 f^{3} 5 d^{5} K_{6}^{\circ}-4 f^{3} 6 p^{5} K_{6}^{0}$ & $10-118$ \\
\hline $5322.77^{*}$ & 0.48 & $4 f^{3} 5 d^{5} L_{6}^{0}-4 f^{3} 6 p^{5} K_{5}^{6}$ & $6-110$ \\
\hline 5681.88 & 1.16 & $4 f^{3} 5 d^{5} H_{5}^{\circ}-4 f^{2} 5 d^{2}{ }^{3} G_{5}$ & $26-134$ \\
\hline $6017.80^{*}$ & 1.11 & $4 f^{3} 5 d^{5} G_{2}^{\circ}-4 f^{3} 6 p^{5} H_{3}^{5}$ & $23-126$ \\
\hline $6165.94^{*}$ & 0.92 & $4 f^{3} 5 d^{5} I_{4}^{\circ}-4 f^{3} 6 p^{5} I_{4}$ & $15-116$ \\
\hline $\begin{array}{c}6656.83^{*} \\
\text { Pr III }\end{array}$ & 1.82 & $4 f^{3} 5 d^{3} L_{9}^{\circ}-4 f^{3} 6 p^{3} K_{8}$ & $60-146$ \\
\hline 4910.82 & 0.17 & $4 f^{3}{ }^{4} I_{11 / 2}^{\circ}-4 f^{2} 5 d^{4} H_{11 / 2}$ & $296-322$ \\
\hline 4929.12 & 0.36 & $4 f^{3}{ }^{4} I_{13 / 2}^{\circ}-4 f^{2} 5 d^{4} H_{13 / 2}$ & $297-325$ \\
\hline 5284.69 & 0.17 & $4 f^{34}{ }^{4} I_{11 / 2}^{\circ}-4 f^{2} 5 d^{4} H_{9 / 2}$ & 296-319 \\
\hline 5299.99 & 0.36 & $4 f^{3}{ }^{4} I_{13 / 2}^{\circ}-4 f^{2} 5 d^{4} H_{11 / 2}^{9 / 2}$ & $297-322$ \\
\hline 5844.41 & 1.24 & $4 f^{32} H_{9 / 2}^{\circ}-4 f^{2} 5 d^{2} G_{7 / 2}$ & $300-332$ \\
\hline 5998.97 & 0.17 & $4 f^{34} I_{11 / 2}^{o}-4 f^{2} 5 d^{4} G_{9 / 2}$ & $296-315$ \\
\hline 6053.00 & 0.00 & $4 f^{34} I_{9 / 2}^{\circ}-4 f^{2} 5 d^{4} G_{7 / 2}$ & 295-311 \\
\hline 6090.01 & 0.36 & $4 f^{3}{ }^{4} I_{13 / 2}^{o}-4 f^{2} 5 d^{4} H_{11 / 2}$ & $297-318$ \\
\hline 6160.23 & 0.17 & $4 f^{3}{ }^{4} I_{11 / 2}^{\circ}-4 f^{2} 5 d^{4} H_{9 / 2}$ & 296-314 \\
\hline 6195.62 & 0.00 & $4 f^{3{ }^{4}} I_{9 / 2}^{\circ}-4 f^{2} 5 d^{4} H_{7 / 2}$ & $295-310$ \\
\hline 6500.04 & 1.72 & $4 f^{32} G_{7 / 2}^{\circ}-4 f^{2} 5 d^{2} F_{5 / 2}$ & $306-334$ \\
\hline 6616.46 & 1.55 & $4 f^{32} H_{11 / 2}^{\circ}-4 f^{2} 5 d^{4} F_{9 / 2}^{5 / 2}$ & $303-332$ \\
\hline 6692.25 & 1.16 & $4 f^{34} F_{3 / 2}^{\circ}-4 f^{2} 5 d^{4} F_{3 / 2}$ & 299-326 \\
\hline 6706.70 & 0.55 & $4 f^{34} I_{15 / 2}^{o}-4 f^{2} 5 d^{2} I_{13 / 2}^{j / 2}$ & $298-318$ \\
\hline
\end{tabular}

${ }^{1}$ Lines used only for solar abundance analysis.

2 The level numbers in the model atom.

${ }^{*}$ HFS is taken into account.

We use homogeneous blanketed model atmospheres. The small grid of models with $T_{\text {eff }}$ ranging between $7500 \mathrm{~K}$ and $9500 \mathrm{~K}$ in step of $500 \mathrm{~K}, \log g=4$, and solar chemical composition was calculated with the MAFAGS code (Fuhrmann et al. 1997), which considers line-blanketing using the opacity distribution functions (ODF). The original ODF tables of Kurucz (1994a) were scaled by -0.16 dex to place the iron opacity calculated by Kurucz with $\log \varepsilon_{\mathrm{Fe}}=7.67$ into correspondence with a value of $\log \varepsilon_{\mathrm{Fe}}=7.51$, which we believe to be the more accurate representation of the solar mixture. We refer to abundances on the usual scale where $\log \varepsilon_{\mathrm{H}}=12$.

For HD $24712\left(T_{\text {eff }}=7250 \mathrm{~K}, \log g=4.3,[\mathrm{Fe} / \mathrm{H}]=0\right.$ according to Ryabchikova et al. 1997), the model atmosphere was computed by Frank Grupp with the MAFAGS-OS code (Grupp 2004), which is based on up-to-date continuous opacities and includes the effects of line-blanketing by means of opacity sampling.

HD 24712 possesses a magnetic field with a mean magneticfield modulus $\langle B\rangle$ that increases from $2.5 \mathrm{kG}$ at magnetic minimum to $\sim 3.1-3.3 \mathrm{kG}$ at magnetic maximum (Ryabchikova et al. 2007b). We ignore the influence of the magnetic field on atmospheric structure, based on the results of Kochukhov et al. (2005) who demonstrated that the difference in temperature and gas pressure distributions between magnetic and non-magnetic model atmospheres with $T_{\text {eff }}=8000 \mathrm{~K}$ does not exceed $30 \mathrm{~K}$ and $6 \%$, respectively, for field strengths smaller than $5 \mathrm{kG}$. However, splitting of the spectral lines in the magnetic field is 
taken into account in LTE abundance analysis. Magnetic spectrum synthesis is performed with the help of the SYNTHMAG code (Kochukhov 2007).

There is observational evidence of non-uniform element distribution in the atmosphere of HD 24712. Neodymium is strongly enhanced in the uppermost atmospheric layers according to Paper I. Ryabchikova et al. (2008) and Ryabchikova (2008) demonstrated that $\mathrm{Ca}, \mathrm{Si}, \mathrm{Cr}, \mathrm{Fe}, \mathrm{Sr}$, and $\mathrm{Ba}$ are concentrated in deep atmospheric layers. One believes that radiatively driven diffusion is one of the main processes responsible for these inhomogeneities. The self-consistent diffusion models predict the vertical distributions of $\mathrm{Mg}, \mathrm{Si}, \mathrm{Ca}, \mathrm{Ti}$, and $\mathrm{Fe}$, which qualitatively reproduce the corresponding element stratifications found empirically for some Ap stars (Alecian \& Stift 2007; LeBlanc \& Monin 2004). However, no theoretical predictions are available for the REE due to an incompleteness of atomic data on energy levels and transition probabilities. Stratified distribution of chemical elements in the atmosphere can influence the atmospheric structure. Modelling chemically non-uniform stellar atmospheres based on abundance gradients determined empirically is in progress (Shulyak 2008, private communication). Preliminary results of the iterative procedure for HD 24714 predict the slight change in the model parameters ( $T_{\text {eff }}=7250 \mathrm{~K}$ and $\left.\log g=4.1\right)$ and lead to only minor changes in our results for the Pr analysis presented in Sect. 6.

\section{Departures from LTE for Pr II-Pr III}

In the line-formation layers of the atmospheres with $T_{\text {eff }}$ between $7250 \mathrm{~K}$ and $8000 \mathrm{~K}$, the number density of Pr II is higher than that of Pr III (see Fig. 4). However, Pr II declines dramatically in hotter atmospheres. Non-LTE calculations indicate that the main non-LTE effect for Pr II is overionization caused by a super-thermal radiation of non-local origin close to the thresholds of the $4 \mathrm{f}^{3} 6 \mathrm{p}$ levels with $E_{\mathrm{exc}}=3 \mathrm{eV}-4 \mathrm{eV}\left(\lambda_{\mathrm{thr}}=1600 \AA\right.$ to $1850 \AA$ ). Photoionization is able to drain the populations of these levels in the model with the lowest temperature, $T_{\text {eff }}=7250 \mathrm{~K}$, and the effect is strengthened as $T_{\text {eff }}$ increases. The population loss is redistributed over many levels, producing overall depletion of the first ionization stage in the line-formation layers. In contrast, photoionization of the Pr III levels is inefficient due to the low stellar fluxes in the far ultraviolet $(\lambda<900 \AA)$, where the ionization edges of the Pr III ground state and the low excitation levels are located. At $T_{\text {eff }} \leq 8000 \mathrm{~K}$, overionization of Pr II leads to an overpopulation of Pr III. At higher temperatures, Pr III represents the state in which the majority of the element exists and preserves the thermodynamic equilibrium (TE) total number density.

We find that the mechanisms driving departures from LTE for Pr II/III are similar in atmospheres with a uniform and stratified distribution of praseodymium, although the magnitude of the effect is different due to the different location of the lineformation layers in a uniform and stratified atmospheres.

\subsection{The atmospheres with uniform distribution of praseodymium}

In the observed spectrum of HD 24712, 14 lines of Pr III are detected with measured equivalent widths, $W_{\lambda}$, of $17 \mathrm{~m} \AA$ or larger. To predict the equivalent widths of the Pr III lines at a detectable level in the model, which represents the atmosphere of HD 24712, we perform calculations with the praseodymium abundance $[\mathrm{Pr} / \mathrm{H}]=3$. This value also characterizes the mean
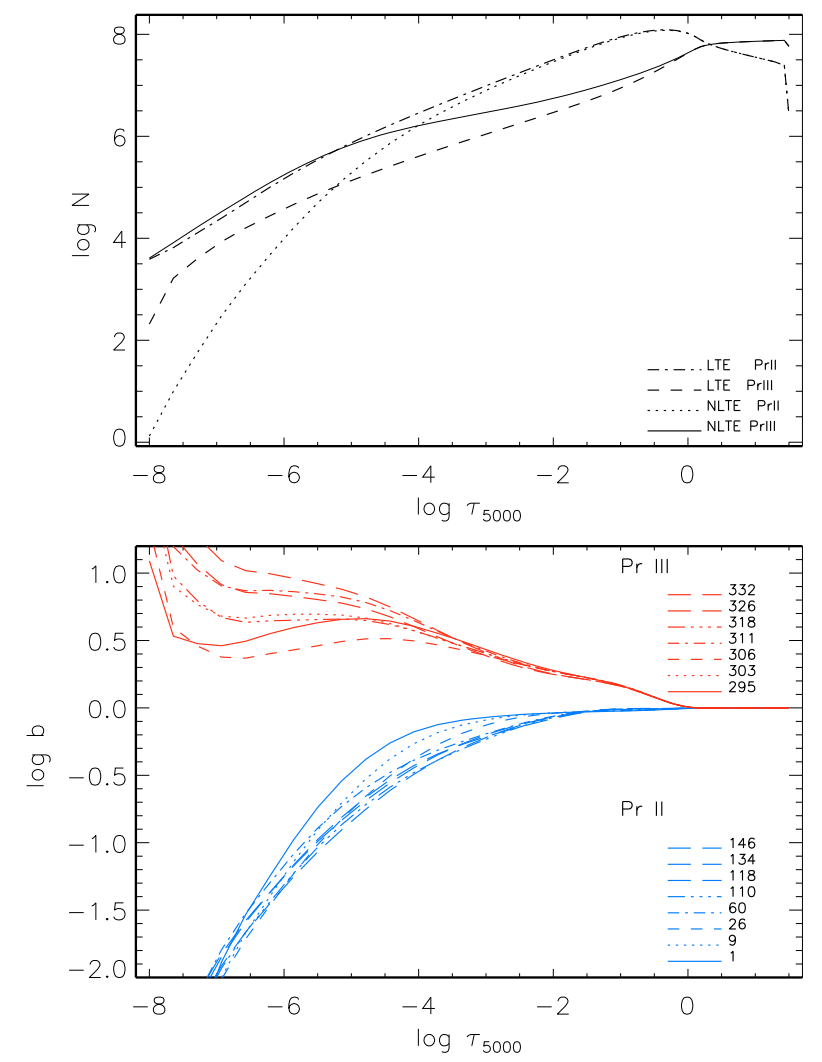

Fig. 4. LTE and non-LTE total number densities of Pr II and Pr III (top panel) and departure coefficients for selected levels of Pr II and Pr III (bottom panel) in the model atmosphere 7250/4.3/0. Successive numbers of the levels in our model atom are quoted. Everywhere in the atmosphere $[\mathrm{Pr} / \mathrm{H}]=3$.

Pr abundance derived from all investigated Pr II and Pr III lines in HD 24712. Figure 4 shows the departure coefficients, $b_{i}=$ $n_{i}^{\mathrm{NLTE}} / n_{i}^{\mathrm{LTE}}$ of the selected levels of Pr II and Pr III as a function of continuum optical depth $\tau_{5000}$ at $\lambda=5000 \AA$ in the model atmosphere with $T_{\text {eff }} / \log g /[\mathrm{M} / \mathrm{H}]=7250 / 4.3 / 0$. Here, $n_{i}^{\mathrm{NLTE}}$ and $n_{i}^{\mathrm{LTE}}$ are the statistical equilibrium and TE (Saha-Boltzmann) number densities, respectively. The Pr II levels with $E_{\text {exc }}<6 \mathrm{eV}$ are strongly coupled with the PrII ground state and to each other inside $\log \tau_{5000} \simeq-1.7$, where their departure coefficients are only slightly below 1 . The outside layers become transparent to the radiation of many Pr II lines arising between lowexcitation terms $\left(E_{\text {exc }}<2 \mathrm{eV}\right)$ and intermediate-excitation terms $\left(E_{\mathrm{exc}}=2.7-4 \mathrm{eV}\right)$. The photon loss in these lines amplifies the underpopulation of the upper levels (level numbers from 110 to 146 in Fig. 4) caused by enhanced photoionization. Pr III is overpopulated in atmospheric layers above $\log \tau_{5000}=0$.

The non-LTE effect on the line strength is determined by the departures from LTE for the lower and upper levels of the transition in the line-formation layers. For the model 7250/4.3/0, the non-LTE effects are small for the PrII lines. The overpopulation of the Pr III levels produces a strengthening of the Pr III lines compared with the LTE case. The theoretical nonLTE and LTE equivalent widths of the selected lines and the non-LTE abundance corrections $\Delta_{\mathrm{NLTE}}=\log \varepsilon_{\mathrm{NLTE}}-\log \varepsilon_{\mathrm{LTE}}$ are presented in Table 4 (Cols. 6-8). Oscillator strengths used in these calculations are taken from Kurucz \& Bell (1995) for the Pr II lines and are computed in this paper for the lines of Pr III. 


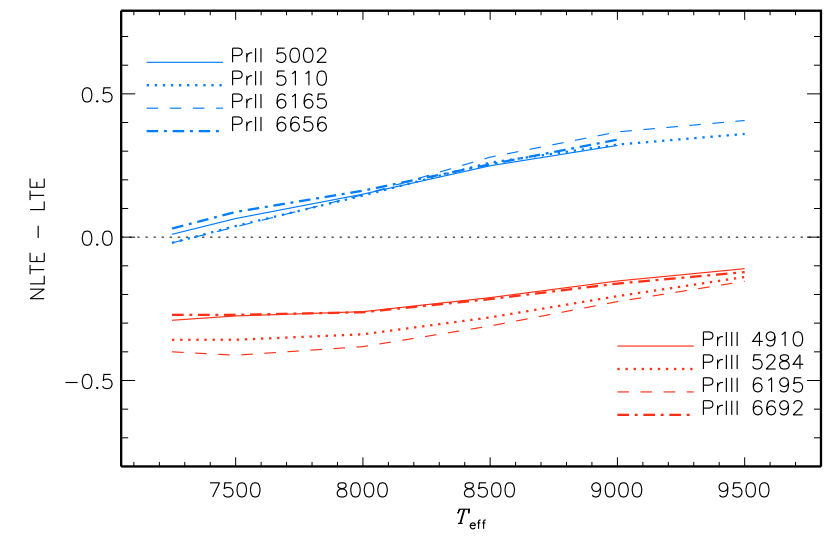

Fig. 5. Non-LTE abundance corrections for the Pr II and Pr III lines depending on $T_{\text {eff. }}$. For all models, $\log g=4$. The calculations were made with the $\operatorname{Pr}$ abundance $[\mathrm{Pr} / \mathrm{H}]=3$.

As expected, for the Pr II lines, the departures from LTE increase with $T_{\text {eff }}$. The higher $T_{\text {eff }}$, the stronger the ultraviolet radiation is, producing the amplified overionization of PrII. Figure 5 shows the calculated non-LTE abundance corrections for some lines of PrII and PrIII depending on $T_{\text {eff }}$. Everywhere, $[\mathrm{Pr} / \mathrm{H}]=3$ is adopted. For the Pr II lines, $\Delta_{\mathrm{NLTE}}$ are positive. They are small at $T_{\text {eff }}=7250 \mathrm{~K}$ and $7500 \mathrm{~K}$ with $\Delta_{\mathrm{NLTE}}<0.1 \mathrm{dex}$, and increase to approximately $0.4 \mathrm{dex}$ at $T_{\text {eff }}=9500 \mathrm{~K}$. In the hottest model, Pr II $\lambda 5002$ and $\lambda 6656$ have $W_{\lambda} \leq 1 \mathrm{~m} \AA$, and their $\Delta_{\text {NLTE }}$ are not shown. In contrast, the departures from LTE for Pr III are smaller toward higher $T_{\text {eff }}$ because doubly-ionized praseodymium tends to represent the state in which the majority of the element exists. The non-LTE abundance corrections are negative for the Pr III lines with $\Delta_{\mathrm{NLTE}} \simeq(-0.3)-(-0.4)$ dex at $T_{\mathrm{eff}}=7500 \mathrm{~K}$ and $\Delta_{\mathrm{NLTE}} \simeq$ -0.1 dex at $T_{\text {eff }}=9500 \mathrm{~K}$.

We also present the revised non-LTE abundance corrections for the Nd II and Nd III lines (Fig. 6). The opacity package of the DETAIL code has been updated by the inclusion of the quasimolecular Lyman $\alpha$ satellites following the implementation by Castelli \& Kurucz (2001) of the Allard et al. (1998) theory and the use of the extended line list based not only on measured but also predicted lines from Kurucz \& Bell (1995). The effect of the increased UV opacity below $1700 \AA$ is detectable only for the lines of $\mathrm{Nd}$ II at $T_{\text {eff }} \geq 8500 \mathrm{~K}$. At lower temperatures, the revised $\Delta_{\mathrm{NLTE}}$ of the NdII lines agree to within $0.01 \mathrm{dex}-$ 0.03 dex with the corresponding values obtained in Paper I. For the Nd III lines, the maximal difference between the revised and Paper's I $\Delta_{\mathrm{NLTE}}$ constitutes $0.05 \mathrm{dex}$ at $T_{\mathrm{eff}}=8500 \mathrm{~K}$, and is smaller at both lower and higher temperatures. Thus, the $\mathrm{Nd}$ abundance distributions in the atmospheres of HD $24712\left(T_{\text {eff }}=\right.$ $7250 \mathrm{~K})$ and $\gamma \mathrm{Equ}\left(T_{\mathrm{eff}}=7700 \mathrm{~K}\right)$ derived in our earlier paper need not be revised. For the Nd II lines at $T_{\text {eff }} \geq 8500 \mathrm{~K}$, we now obtain smaller departures from LTE due to lower amounts of ionizing radiation. The difference in $\Delta_{\text {NLTE }}$ equals between 0.1 dex and 0.2 dex for different lines at different temperatures.

\subsection{The atmospheres with stratified distribution of praseodymium}

Departures from LTE for Pr II/III are investigated in the model 7250/4.3/0 with the stratified Pr abundance distribution determined in Sect. 6.2 and shown by continuous curve in the

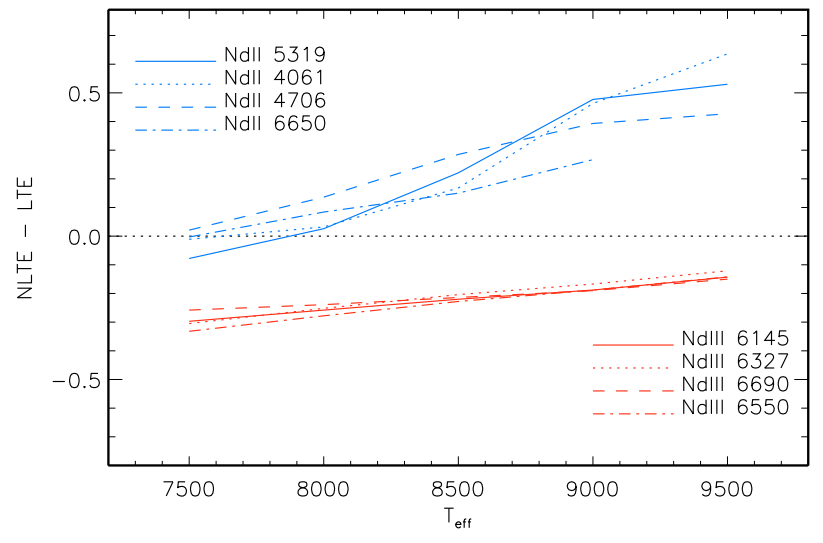

Fig. 6. Non-LTE abundance corrections for the Nd II and Nd III lines depending on $T_{\text {eff. }}$ For all models, $\log g=4$. The calculations were made with the $\mathrm{Nd}$ abundance $[\mathrm{Nd} / \mathrm{H}]=2.5$.
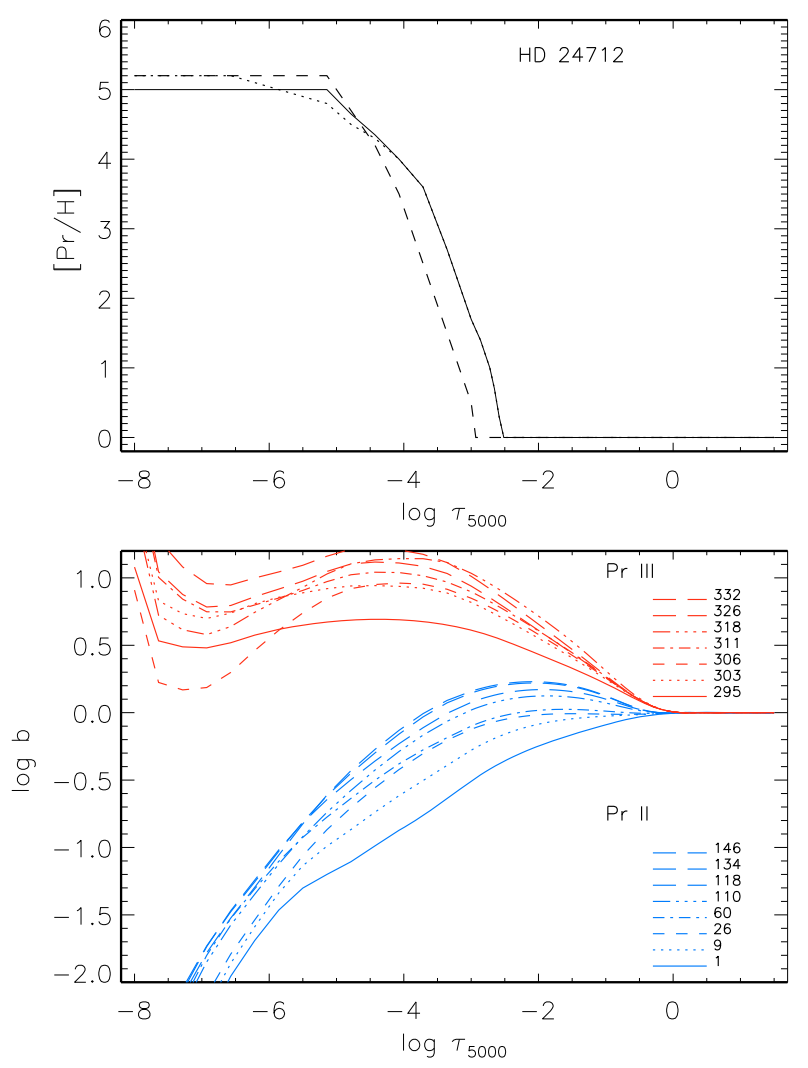

Fig. 7. Top panel: the $[\mathrm{Pr} / \mathrm{H}]$ ratio distributions found in the atmosphere of HD 24712 (continuous line) and from the test calculations with 2 dex smaller photoionization cross-sections (dashed line) and with a variable effective collision strength for forbidden transitions (dotted line). Bottom panel: the departure coefficients $\log b$ for the selected levels of PrII and PrIII in the model 7250/4.3/0 representing the atmosphere of HD 24712 from calculations with the praseodymium distribution shown in the top panel by continuous curve. See text for more details.

top panel of Fig. 7. The departure coefficients for the selected levels of Pr II and Pr III are shown in the bottom panel of the same figure. The theoretical non-LTE and LTE equivalent widths together with non-LTE abundance corrections are given in Table 4 (Cols. 9-11). 
Table 3. Solar praseodymium abundance from calculations with the ATLAS9 solar model atmosphere.

\begin{tabular}{|c|c|c|c|c|c|c|c|}
\hline \multirow[t]{2}{*}{$\lambda[\AA]$} & \multicolumn{3}{|c|}{$\log g f$} & \multicolumn{3}{|c|}{$\log (\operatorname{Pr} / \mathrm{H})_{\text {Lte }}$} & \multirow[t]{2}{*}{$\Delta_{\mathrm{NLTE}}$} \\
\hline & $\mathrm{KB}^{1}$ & ILW $^{2}$ & $\mathrm{LCH}^{3}$ & KB & ILW & $\mathrm{LCH}$ & \\
\hline 4222.93 & 0.13 & 0.27 & 0.24 & -11.14 & -11.28 & -11.25 & +0.03 \\
\hline 4449.83 & -0.32 & -0.17 & -0.26 & -11.07 & -11.22 & -11.13 & +0.02 \\
\hline 5259.73 & 0.08 & 0.11 & 0.07 & -11.11 & -11.14 & -11.10 & +0.04 \\
\hline 5322.77 & -0.32 & -0.32 & -0.12 & -11.04 & -11.04 & -11.24 & +0.02 \\
\hline & \multicolumn{3}{|c|}{ LTE-average } & $\begin{array}{l}-11.09 \pm 0.04 \\
-11.06 \pm 0.04\end{array}$ & $\begin{array}{l}-11.17 \pm 0.10 \\
-11.14 \pm 0.10\end{array}$ & $\begin{array}{l}-11.18 \pm 0.08 \\
-11.15 \pm 0.08\end{array}$ & \\
\hline
\end{tabular}

${ }^{1}$ Kurucz \& Bell $(1995, \mathrm{~KB}) ;{ }^{2}$ Ivarsson et al. $(2001, \mathrm{ILW}) ;{ }^{3}$ Li et al. $(2007, \mathrm{LCH})$.

Non-LTE calculations show the depletion of Pr II and enhanced number density of Pr III in the line formation layers similar to the case of homogeneous Pr abundance distribution. However, three important distinctions in the behavior of departure coefficients can be seen.

- The magnitude of the depletion of Pr II is much larger in the stratified atmosphere, in particular, above $\log \tau_{5000}=-4$ where the lines are formed.

- In the stratified atmosphere, for every pair of the Pr II levels with $i<j$, an inequality $b_{i}<b_{j}$ is valid.

- Above $\log \tau_{5000}=-4$ in the stratified atmosphere, the excited levels of Pr III are decoupled from the ground state to a larger extent than in the case of the homogeneous Pr abundance distribution.

In the stratified atmosphere, the Pr lines are formed in the uppermost atmospheric layers, above $\log \tau_{5000}=-4$, where departures from LTE are significant. Non-LTE causes a weakening of the Pr II lines due to a decrease in the number of absorbers $\left(b_{i}<1\right)$ and the fact that the line source function $S_{i j} \simeq b_{j} / b_{i} B_{v}\left(T_{\mathrm{e}}\right)$ exceeds the Planck function $\left(b_{i}<b_{j}\right)$. The non-LTE abundance corrections for various Pr II lines are positive and may reach +1.2 dex. The Pr III lines are stronger than in the LTE case, and $\Delta_{\text {NLTE }}$ may reach -0.7 dex. Since the non-LTE effects have the opposite sign for the Pr II and Pr III lines, they are important to the comparison of Pr abundances derived from these lines.

\section{Solar abundance of praseodymium}

We first apply the non-LTE calculations to the Pr abundance analysis for the solar atmosphere. Earlier determinations were performed by Biémont et al. (1979) based on the nine lines of Pr II in the 3990-5330 $\AA$ spectral region and by Ivarsson et al. (2003) based on the three lines of PrII. The derived mean abundances of praseodymium were surprisingly different, $\log (\operatorname{Pr} / \mathrm{H})_{\odot}=-11.29 \pm 0.08$ and $\log (\operatorname{Pr} / \mathrm{H})_{\odot}=-11.60 \pm 0.1$, respectively. The study by Ivarsson et al. (2003) was based on the modern accurate laboratory measurements for oscillator strengths of Ivarsson et al. (2001). Ivarsson et al. (2003) also tested the influence of 3D effects on the determination of the Pr abundance and drew a preliminary conclusion that the differences between the equivalent widths of the Pr II lines derived for the $3 \mathrm{D}$ and $1 \mathrm{D}$ cases were insignificant.

Each of the lines used by our predecessors was checked for blending using the NSO solar flux spectrum (Kurucz et al. 1984) observed with the spectral resolving power $R \simeq 340000$ at wavelengths between $4000 \AA$ and $4700 \AA$ and with $R=520000$ at longer wavelengths. We find four lines suitable for precise spectral fitting, which are listed in Table 3 . The spectral region around each investigated line was synthesized with the SIU code
(Reetz 1991). For a given atomic level, sIU computes the nonLTE population as the production of the LTE occupation number and the corresponding departure coefficient. In LTE calculations, the revised Pr II partition function is applied. Calculations of the Pr II lines were made with three different sets of transition probabilities taken from Kurucz \& Bell (1995), Ivarsson et al. (2001), and Li et al. (2007). Atomic parameters for other atomic lines in the synthesized regions were taken from the latest release of VALD (Kupka et al. 1999). For molecular lines, we applied the data compiled by Kurucz (1994b). We used the theoretical ATLAS9 model atmosphere of the Sun (5777/4.44/0, Heiter et al. 2002) with convection treated according to Canuto et al. (1996) and the semi-empirical model of Holweger \& Müller (1974, HM). A microturbulence velocity $V_{\text {mic }}=0.9 \mathrm{~km} \mathrm{~s}^{-1}$ was adopted. Our synthetic flux profiles were convolved with a profile that combined a rotational broadening of $1.8 \mathrm{~km} \mathrm{~s}^{-1}$, broadening by macroturbulence with a radial-tangential profile of $V_{\mathrm{mac}}=3.6 \mathrm{~km} \mathrm{~s}^{-1}$, and instrumental broadening with a Gaussian profile corresponding to the spectral resolution. A macroturbulence velocity was allowed to vary within $0.2 \mathrm{~km} \mathrm{~s}^{-1}$ to derive the best-fit solution for the observed line shape.

The best-fit solutions for the observed solar line profiles are shown in Fig. 8. The results from calculations with the ATLAS9 solar model atmosphere are presented in Table 3. Below we present brief notes for the individual lines.

Pr II $4222.93 \AA$. The continuum flux at $4222.93 \AA$ is influenced by the far wing of the Ca I resonance line at $4226.7 \AA$. The Ca I line is treated using the measured oscillator strength from Smith \& Gallagher (1966) and the van der Waals broadening parameters based on the advanced perturbation theory of Anstee \& O'Mara (1995). The Pr II $4222.93 \AA$ line ovelapps partially the blue wing of the spectral feature produced by two $\mathrm{CH}$ lines at $4223.091 \AA$ and $4223.113 \AA$. A half-width of this spectral feature is only slightly affected by the Pr II line and is fitted reasonably well if we reduce oscillator strengths for both $\mathrm{CH}$ lines by 0.3 dex from the values given by Kurucz (1994b). Small changes in wavelengths (no more than $0.014 \AA$ ) and oscillator strengths (no more than $0.27 \mathrm{dex}$ ) were introduced in fitting the CrI $4222.740 \AA$ and Fe I $4223.237 \AA$ lines to obtain a more accurate fit.

Pr II $5259.7 \AA$. This line is well isolated and fitted well by spectrum synthesis. The Ni I $5259.466 \AA$ and Ti I $5259.973 \AA$ lines shown in Fig. 8 do not affect the Pr abundance determination. For the Ni I line, changes in wavelength of $0.011 \AA$ and in oscillator strength of -0.4 dex were applied in fitting the line profile, while a $0.005 \AA$ wavelength change was required for the Ti I line.

$\underline{\text { Pr II } 5322.8 \AA}$ is also well isolated, but its local continuum flux is influenced by the far wing of the Fe I $5324.179 \AA$ line. 

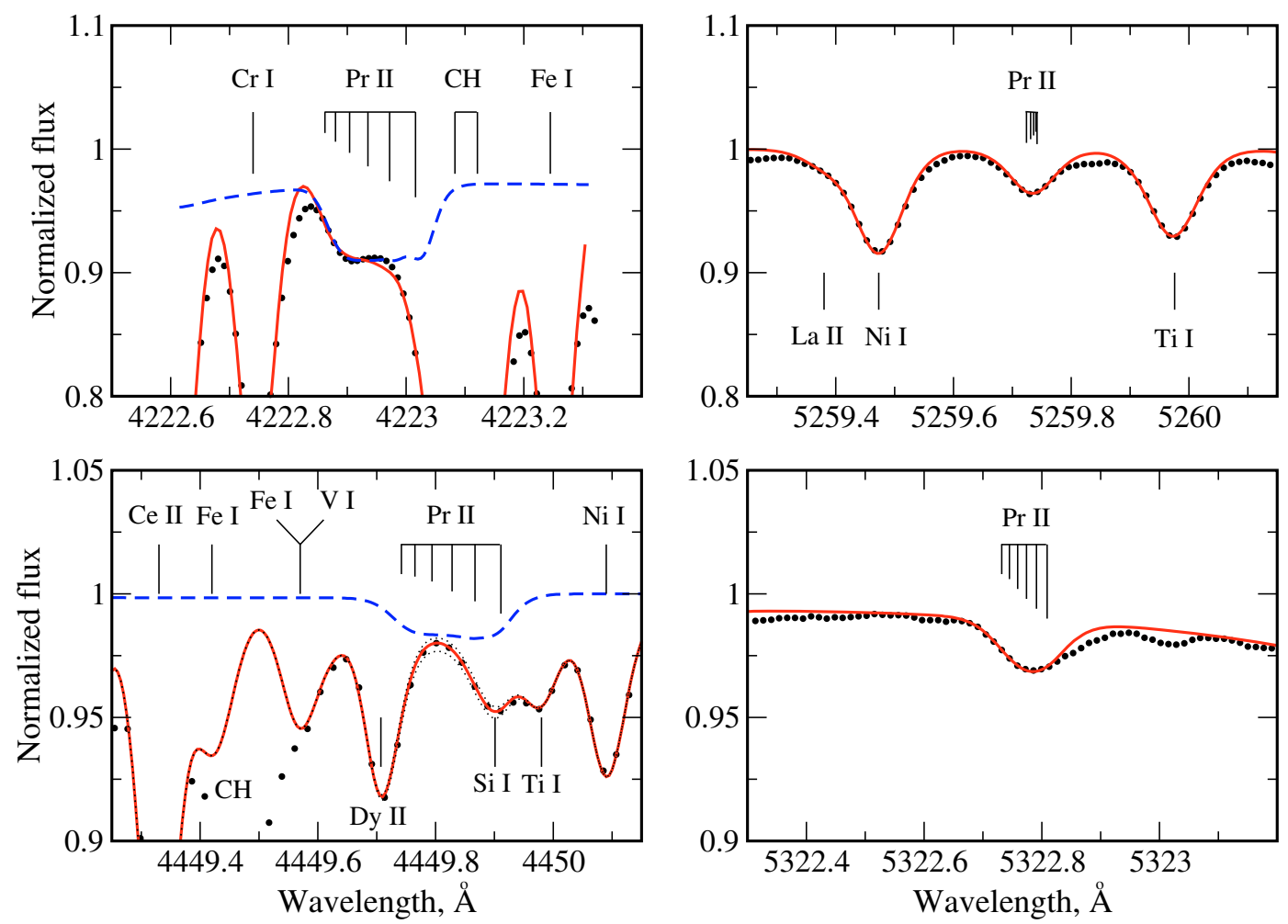

Fig. 8. Synthetic spectra (continuous curve) and pure Pr II line profiles (dashed curve, for $\lambda 4223 \AA$ and $\lambda 4449 \AA$ lines only) calculated with the ATLAS9 solar model atmosphere in comparison with the observed NSO solar flux spectrum (Kurucz et al. 1984, bold dots). For the $4449 \AA$ A blend, dotted curves show the effect on the synthetic spectrum of a 0.05 dex variation in the adopted Pr abundance. The Pr II lines are treated based on non-LTE line formation.

With the most accurately determined atomic parameters of the Fe I line, $\log g f=-0.103$ (Bard et al. 1991) and $\log \gamma_{6} / N_{\mathrm{H}}=$ -7.035 at $T_{\mathrm{e}}=10000 \mathrm{~K}$ (increased by 0.2 dex compared with the value of Anstee \& O'Mara's 1995), the predicted synthetic flux level was still $0.2 \%$ higher than the observed flux level around $5323 \AA$. The local continuum level was, therefore, adjusted to fit the blue wing of Fe I $5324 \AA$. A variation of $0.2 \%$ in the continuum flux produces a 0.05 dex change in the Pr abundance derived from Pr II $5322.8 \AA$.

Pr II $4449.83 \AA$ is located between the Dy II $4449.70 \AA$ and Si I $4449.90 \AA$ lines. The blue wing and the core of the Dy II line are slightly affected by the adjacent lines and well fitted using a centre line wavelength of $4449.707 \AA$, accurate experimental oscillator strength of $\log g f=-1.03$ (Wickliffe et al. 2000), and solar Dy abundance of $\log \varepsilon_{\text {Dy, } \odot}=1.14$ (Lodders 2003). The $\mathrm{Si}$ I line is predicted, and its oscillator strength has to be reduced to $\log g f=-3.33$ to reproduce as well as possible the line core, which is only slightly affected by the HFS components of the Pr II line. In Fig. 8, we show the best fit of the $4449 \AA$ blend and also the synthetic spectra computed with a 0.05 dex change in adopted Pr abundance. The uncertainty in the element abundance derived from Pr II 4449, obviously, does not exceed 0.05 dex.

In the solar atmosphere, the ground state and low excitation levels in Pr II preserve the TE number density. Our nonLTE calculations illustrate that photon pumping enhances the excitation of the upper levels resulting in weakening the lines of interest in comparison with the LTE case. We find that the statistical equilibrium of Pr II in the solar atmosphere is sensitive to a variation in collision excitation rates that include, for the Sun, interactions not only with electrons but also neutral hydrogen atoms. For hydrogenic collisions, we use the formula of
Steenbock \& Holweger (1984) for allowed transitions and follow Takeda (1994) for forbidden transitions. Both theoretical approximations provide only an order of magnitude estimate. The efficiency of hydrogenic collisions in the SE of atoms is represented by a scaling factor $S_{\mathrm{H}}$ applied to the aforementioned formula. Empirical estimates of $S_{\mathrm{H}}$ lie between $S_{\mathrm{H}}=0.002$ and $S_{\mathrm{H}}=1$ (for a review, see Mashonkina 2008). When pure electronic collisions are taken into account $\left(S_{\mathrm{H}}=0\right)$, the nonLTE abundance corrections constitute between +0.03 dex and +0.08 dex for different lines. The non-LTE effects become negligible $\left(\Delta_{\mathrm{NLTE}} \leq 0.01 \mathrm{dex}\right)$ when both types of collisions are included and $S_{\mathrm{H}}=1$. We adopt an intermediate value, $S_{\mathrm{H}}=0.1$. The corresponding non-LTE corrections are shown in the last column of Table 3 .

The average LTE and non-LTE praseodymium abundances from calculations with the ATLAS9 solar model atmosphere and their standard deviations are presented in two bottom strings of Table 3. The Pr abundance determined with the HM model is, on average, higher by 0.05 dex. We note that the statistical error in our abundance determination estimated from the line-to-line scatter corresponds, in fact, to the quoted errors of log $g f$ measurements, which varies between $0.03 \mathrm{dex}$ and $0.07 \mathrm{dex}$ for the lines in Table 3 . The three solutions found with $\log g f$ from different sources are consistent to within the error bars. However, the preference should be given to the ILW and LCH abundances based on the modern experimental transition probabilities. The average ILW and LCH abundances agree to within 0.01 dex. For each set of atomic parameters and for both solar model atmospheres, the derived Pr abundance is between 0.09 dex and 0.23 dex higher than that obtained by Biémont et al. (1979) and between 0.40 dex and 0.54 dex higher than that of Ivarsson et al. (2003). Different studies determined a meteoritic CI Chondrites 
Table 4. Non-LTE and LTE equivalent widths of the Pr II and Pr III lines calculated for the model 7250/4.3/0 with the homogeneous $([\operatorname{Pr} / \mathrm{H}]=3)$ and stratified element distributions compared to observations ( $W_{\mathrm{obs}}$ ) of HD 24712.

\begin{tabular}{|c|c|c|c|c|c|c|c|c|c|c|c|c|}
\hline \multirow[b]{2}{*}{$\begin{array}{l}\lambda, \\
\AA\end{array}$} & \multirow[b]{2}{*}{$\begin{array}{c}E_{\text {low }}, \\
\mathrm{eV}\end{array}$} & \multirow[b]{2}{*}{$\log g f$} & \multirow[b]{2}{*}{$\begin{array}{r}W_{\text {obs }} \\
\mathrm{m} \AA \\
\end{array}$} & \multirow[b]{2}{*}{$\begin{array}{l}V_{\text {mic }}, \\
\mathrm{km} \mathrm{s}^{-1}\end{array}$} & \multicolumn{3}{|c|}{$[\mathrm{Pr} / \mathrm{H}]=3$} & \multicolumn{5}{|c|}{ Stratified distribution ${ }^{*}$} \\
\hline & & & & & $W_{\mathrm{LTE}}$ & $W_{\text {NLTE }}$ & $\Delta_{\mathrm{NLTE}}$ & $W_{\mathrm{LTE}}$ & $W_{\text {NLTE }}$ & $\Delta_{\mathrm{NLTE}}$ & $\begin{array}{c}\log \tau_{5000}^{l} \\
\text { NLTE }\end{array}$ & $\begin{array}{c}\log \tau_{5000}^{l+c} \\
\text { NLTE }\end{array}$ \\
\hline 1 & 2 & 3 & 4 & 5 & 6 & 7 & 8 & 9 & 10 & 11 & 12 & 13 \\
\hline Pr II & & & & & & & & & & & & \\
\hline 5002.44 & 0.80 & $-0.87^{1}$ & 12 & 1.0 & 31 & 30 & 0.02 & 40 & 6 & 1.12 & -4.50 & -0.65 \\
\hline 5110.76 & 1.15 & $0.32^{2}$ & 30 & 1.0 & 95 & 96 & -0.02 & 114 & 36 & 1.11 & -4.57 & -1.86 \\
\hline 5129.54 & 0.65 & $-0.13^{1}$ & 26 & 1.0 & 77 & 78 & -0.03 & 94 & 32 & 1.17 & -4.68 & -2.08 \\
\hline 5135.14 & 0.95 & $0.01^{3}$ & 16 & 1.0 & 80 & 80 & -0.01 & 99 & 31 & 1.07 & -4.59 & -1.47 \\
\hline 5292.62 & 0.65 & $-0.26^{3}$ & 23 & 1.0 & 84 & 84 & 0.00 & 108 & 28 & 1.19 & -4.60 & -1.64 \\
\hline 5322.77 & 0.48 & $-0.32^{3}$ & 22 & 1.0 & 99 & 100 & -0.01 & 127 & 36 & 1.18 & -4.64 & -1.44 \\
\hline 5681.88 & 1.16 & $-0.60^{2}$ & 10 & 1.0 & 34 & 33 & 0.02 & 42 & 8 & 1.02 & -4.49 & -0.75 \\
\hline 6017.80 & 1.11 & $-0.26^{2}$ & 20 & 1.0 & 69 & 70 & -0.02 & 93 & 17 & 1.10 & -4.48 & -1.03 \\
\hline 6165.94 & 0.92 & $-0.20^{2}$ & 17 & 1.0 & 92 & 93 & -0.02 & 126 & 27 & 1.13 & -4.55 & -1.38 \\
\hline $\begin{array}{c}6656.83 \\
\text { Pr III }\end{array}$ & 1.82 & $0.08^{2}$ & 14 & 1.0 & 41 & 39 & 0.03 & 53 & 9 & 1.06 & -4.47 & -1.19 \\
\hline 4910.82 & 0.17 & $-1.95^{4}$ & 37 & 1.7 & 10 & 17 & -0.29 & 22 & 48 & -0.51 & -5.20 & -2.65 \\
\hline 4929.12 & 0.36 & -2.07 & 23 & 1.0 & 6 & 11 & -0.27 & 12 & 32 & -0.61 & -5.17 & -2.06 \\
\hline 5284.69 & 0.17 & -0.77 & 92 & 1.0 & 48 & 60 & -0.36 & 68 & 83 & -0.48 & -6.31 & -5.74 \\
\hline 5299.99 & 0.36 & -0.72 & 94 & 1.05 & 44 & 58 & -0.36 & 65 & 81 & -0.48 & -6.24 & -5.66 \\
\hline 5844.41 & 1.24 & -1.01 & 48 & 1.0 & 13 & 20 & -0.24 & 21 & 48 & -0.62 & -5.45 & -4.02 \\
\hline 5998.97 & 0.17 & -1.87 & 63 & 1.55 & 14 & 24 & -0.29 & 33 & 61 & -0.45 & -5.47 & -3.81 \\
\hline $6-053.00$ & 0.00 & -1.98 & 70 & 1.55 & 14 & 24 & -0.29 & 37 & 64 & -0.42 & -5.44 & -3.92 \\
\hline 6090.01 & 0.36 & -0.87 & 94 & 1.1 & 46 & 61 & -0.36 & 71 & 91 & -0.53 & -6.19 & -5.58 \\
\hline 6160.23 & 0.17 & -1.02 & 96 & 1.1 & 43 & 59 & -0.37 & 72 & 88 & -0.43 & -6.23 & -5.69 \\
\hline 6195.62 & 0.00 & -1.07 & 94 & 1.0 & 46 & 63 & -0.40 & 74 & 94 & -0.57 & -6.19 & -5.63 \\
\hline 6500.04 & 1.72 & -1.26 & 25 & 1.0 & 3 & 5 & -0.26 & 5 & 22 & -0.70 & -5.11 & -2.13 \\
\hline 6616.46 & 1.55 & -1.50 & 25 & 1.0 & 3 & 5 & -0.25 & 5 & 20 & -0.69 & -5.07 & -1.92 \\
\hline 6692.25 & 1.16 & -2.11 & 17 & 1.0 & 2 & 3 & -0.27 & 3 & 15 & -0.73 & -5.02 & -1.14 \\
\hline 6706.70 & 0.55 & -1.49 & 41 & 1.5 & 18 & 30 & -0.31 & 38 & 75 & -0.58 & -5.49 & -4.22 \\
\hline
\end{tabular}

${ }^{1}$ Li et al. (2007); ${ }^{2}$ Kurucz \& Bell (1995); ${ }^{3}$ Ivarsson et al. (2001); ${ }^{4}$ this study, for all Pr III lines; ${ }^{*}$ shown by a continuous curve in the top panel of Fig. 7.

abundance $\log (\operatorname{Pr} / \mathrm{H})_{\text {met }}=-11.20 \pm 0.04$ (Grevesse et al. 1996; Palme \& Jones 2005), $-11.22 \pm 0.03$ (Lodders 2003), or $-11.25 \pm 0.03$ (Asplund et al. 2005). The solar Pr abundance determined in this study for the ATLAS9/HM model, $\log (\operatorname{Pr} / \mathrm{H})_{\odot}=$ $-11.15 \pm 0.08 /-11.10 \pm 0.08$, exceeds the meteoritic values by between 0.05 dex and 0.10 dex.

In solar-abundance determinations, we use a partition function calculated by its definition and based on an extensive set of the predicted energy levels of Pr II, $U_{\text {calc }}(\operatorname{Pr}$ II). As can be seen from Table 1, $U_{\text {calc }}(\operatorname{Pr}$ II) is about $30 \%$ higher than that for the experimental energy levels only, $U_{\exp }(\operatorname{Pr}$ II), at temperatures of the line formation layers in the solar atmosphere.

With $U_{\exp }(\operatorname{Pr} I \mathrm{I})$, the inferred solar $\operatorname{Pr}$ abundance is $\log (\operatorname{Pr} / \mathrm{H})_{\odot}=-11.22$ and -11.17 , for the ATLAS9 and the Holweger \& Müller (1974) models, respectively. Thus, the accurate determination of solar Pr abundance depends on the completeness of known Pr II levels and the accuracy of their energies. It appears that the current theoretical energy calculations provide us with an upper limit for the solar Pr abundance.

\section{Praseodymium in the roAp star HD 24712}

We study the Pr abundance in the atmosphere of the roAp star HD 24712 using its average spectrum obtained during spectroscopic monitoring on November 11/12, 2004 with the UVES spectrograph at the 8.2-m telescope, UT2 (Kueyen), of the VLT on Paranal, Chile (programme 274.D-5011). The observations and data reduction were described by Ryabchikova et al. (2007b). The spectrum was observed close to magnetic maximum, which coincides roughly with the REE spectral line intensity maximum. The surface magnetic field measured at the magnetic maximum was $\langle B\rangle=3.1-3.3 \mathrm{kG}$ (Ryabchikova et al. 2007b).

\subsection{The problem of the Pr abundance in HD24 712}

For the beginning, we determine an element abundance using the lines of Pr II and Pr III listed in Table 4 and assuming the uniform distribution of Pr in the atmosphere of HD 24712. The choice of the spectral lines was determined mainly by the possibility of pulsation measurements in the HD 24712 spectrum. Magnetic spectrum synthesis is performed with the help of the SYNTHMAG code for the assumption of LTE and using the model atmosphere 7250/4.3/0. We apply the most recent experimental transition probabilities measured by Ivarsson et al. (2001) and Li et al. (2007) if available and oscillator strengths from Kurucz \& Bell (1995) for the remaining Pr II lines. The derived LTE abundance was $\log (\operatorname{Pr} / \mathrm{H})=-9.4 \pm 0.2$ for 10 Pr II lines and 2.1 dex higher at $\log (\operatorname{Pr} / \mathrm{H})=-7.3 \pm 0.3$, for 14 Pr III lines. This significant discrepancy between the rare-earth 
LTE abundances derived from two ionization stages is typically found for the roAp and cool Ap stars (the REE anomaly, see Ryabchikova et al. 2004).

Our non-LTE code does not include the magnetic field in statistical-equilibrium calculations. A magnetic field should not cause significant changes in the derived level populations, for moderate field strengths. As shown in Sect. 4, departures from LTE for the level populations are caused mainly by UV continua and strong UV transitions. The change in emergent continuum flux around $2000 \AA$ due to the influence of the magnetic field on atmospheric structure does not exceed $7 \%$, for field strengths up to $10 \mathrm{kG}$ (Kochukhov et al. 2005). The critical strong UV transitions have small Zeeman splittings, since the splittings scale with the wavelength. Further abundance determinations are, therefore, performed in the field-free approximation.

With fixed level populations, the magnetic field can affect an abundance analysis by Zeeman splitting of spectral lines. Therefore, we investigated the possibility of replacing magnetic broadening effects with other broadening mechanisms, such as a microturbulence. Different spectral lines used in this study have different Zeeman patterns. The Pr II lines are weak and HFS-affected. We adopted a common value, $V_{\text {mic }}=1 \mathrm{~km} \mathrm{~s}^{-1}$, in their analysis. For each line of Pr III, a pseudo-microturbulence velocity was calculated as follows. We first attempted to fit the line profile with the SYNTHMAG code to derive the Pr abundance. Then, calculations were made with the SYNTH3 code (Kochukhov 2007), which ignores the existence of a magnetic field. The element abundance was fixed and $V_{\text {mic }}$ varied until the theoretical equivalent width reached its observed value. The individual values of $V_{\text {mic }}$ are given in Table 4. Applying this procedure we find only minor changes in the element abundances of 0.14 dex and 0.16 dex for the Pr II and Pr III lines, respectively, compared with those determined by magnetic spectrum synthesis. We note that the difference in the abundances derived for the two ionization stages remained almost the same.

Next, we checked whether the departures from LTE could account for the problem in determining the praseodymium abundance in HD 24712 and found that non-LTE tends to reduce the difference between the LTE abundances but failed to remove the difference completely. The non-LTE abundance was obtained to be $\log (\operatorname{Pr} / \mathrm{H})=-9.22 \pm 0.19$ from the Pr II lines, and $1.90 \mathrm{dex}$ higher when determined from the lines of Pr III. Thus, we were unable to derive consistent abundances of praseodymium from lines of two ionization stages assuming the uniform element distribution in the atmosphere of HD 24712, by either considering non-LTE line formation or taking into account the splitting of spectral lines by the magnetic field. We, therefore, suggest, that as for neodymium (Paper I), praseodymium is distributed nonuniformly in the atmosphere of HD 24 712, although the properties of the $\mathrm{Nd}$ and Pr distributions may differ.

\subsection{Vertical distribution of Pr in the atmosphere}

Using the simplified step-function approximation as an initial estimate of the Pr abundance profile, we modify it with a trial-anderror method based on non-LTE line formation by trying to fit the observed equivalent widths of both Pr II and Pr III lines in the spectrum of HD 24 712. Due to certain limitations of the present non-LTE calculations, mainly due to ignoring Zeeman splitting, we were unable to use the observed line profiles in stratification analysis as completed, for instance, in a LTE analysis of $\mathrm{Ca}$ (Ryabchikova et al. 2008). The final solution is shown in the top panel of Fig. 7. The praseodymium is concentrated mostly

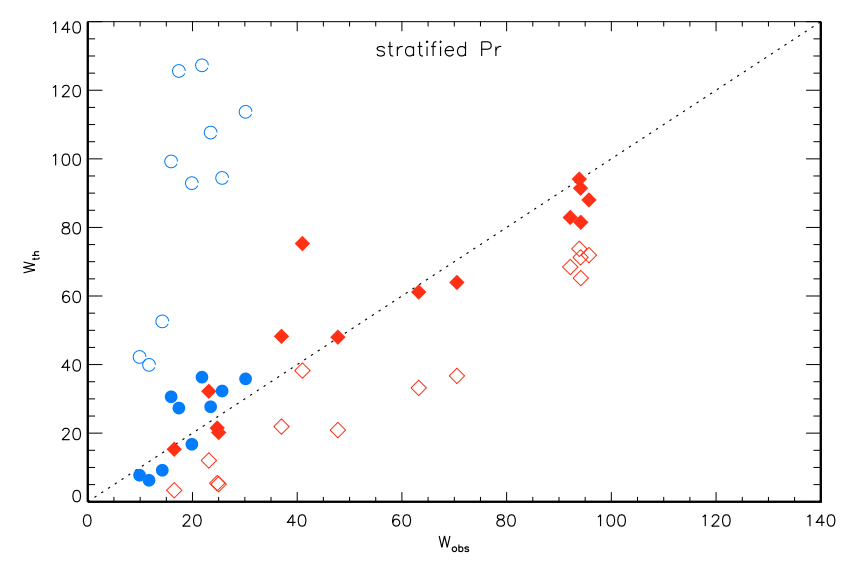

Fig. 9. Observed equivalent widths of the Pr II (circles) and Pr III (diamonds) lines in HD 24712 compared with the theoretical non-LTE (filled symbols) and LTE (open symbols) equivalent widths for the stratified Pr distribution shown in the top panel of Fig. 7 by continuous curve.

above $\log \tau_{5000}=-4$, and the obtained Pr abundance in the layer is $[\mathrm{Pr} / \mathrm{H}] \geq 4$. Table 4 (Col. 10) presents the corresponding nonLTE equivalent widths of the Pr II and Pr III lines. For comparison, we also provide the LTE equivalent widths and non-LTE abundance corrections calculated for the derived Pr distribution. It can be seen that the net non-LTE correction to the Pr abundance $\Delta_{\text {NLTE }}(\operatorname{Pr} I I)-\Delta_{\text {NLTE }}(\operatorname{Pr}$ III) is at the level of $1.8 \mathrm{dex}$, for the stratified atmosphere. To ensure that the LTE element abundances from the lines of Pr II and Pr III are consistent, the layer of enhanced praseodymium must be located above the uppermost depth point in our model, $\log \tau_{5000}=-8$ (!). This implies that any stratification analysis of praseodymium in roAp stars must be performed based on non-LTE line formation. It is worth noting that the location of the enriched praseodymium layer is in fact similar to that found for the neodymium in our earlier study.

A quality of the fit to the observed praseodymium lines in HD 24712 with the derived Pr stratification profile is illustrated in Figs. 9 and 10. The synthetic flux profiles were convolved with the instrumental profile (spectral resolution $R=80000$ ), projected rotational velocity $v_{\mathrm{e}} \sin i=5.6 \mathrm{~km} \mathrm{~s}^{-1}$, and additional broadening parameter equivalent to radial-tangential profile of $V_{\mathrm{mac}}=4 \mathrm{~km} \mathrm{~s}^{-1}$ for the Pr II and $6 \mathrm{~km} \mathrm{~s}^{-1}$ for the Pr III lines. One cannot expect to derive the same quality of fits for the magnetic star with a complex chemically stratified atmosphere as for the Sun. Nevertheless, our non-LTE stratification analysis allows us to reproduce well the observed Pr II and Pr III lines in HD 24712, in contrast to the uniform distribution of Pr. In the latter case, we attempt to reproduce the Pr III $5300 \AA$ and $6195 \AA$ profiles (dashed curves in Fig. 10) with the Pr abundance determined from the non-LTE analysis of the Pr III lines only, $\log (\operatorname{Pr} / \mathrm{H})=-7.32$, but calculate extremely strong lines of $\operatorname{Pr}$ II compared with the observations.

Knowledge of the Pr abundance distribution is important in studying atmospheric pulsations. Figure 11 shows a distribution of the pulsation RV amplitudes and phases of the Pr lines as well as of the lines of Nd II and Nd III and of the H $\alpha$ core in the atmosphere of HD 24712 . The RV data are taken from Ryabchikova et al. (2007b, Online Table 4). We use the average optical depths of line formation, which are calculated with the element abundance distributions obtained in this study for Pr and in Paper I for $\mathrm{Nd}$. We follow the formalism suggested by Achmad et al. (1991) by using the contribution function to the emergent line 

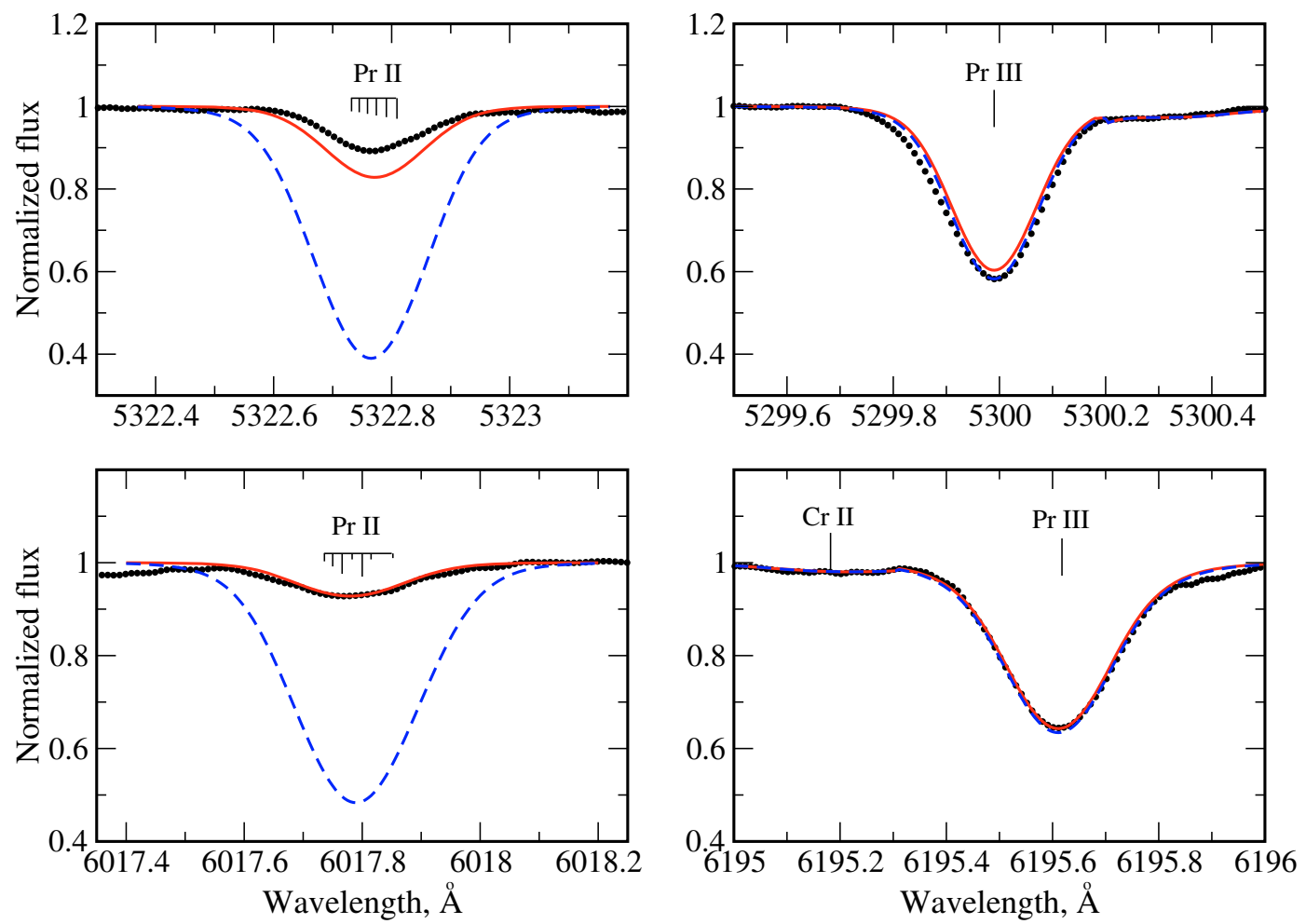

Fig. 10. A comparison between the observed (bold dots) and non-LTE spectra for the selected Pr II and Pr III lines in HD 24712. The non-LTE profiles from calculations with the derived Pr distribution are shown by continuous curves. Dashed curves correspond to the non-LTE profiles from calculations with $\log (\operatorname{Pr} / \mathrm{H})=-7.32$ everywhere in the atmosphere.

radiation. The computed values of $\log \tau_{5000}^{l}$ are given in Table 4 (Col. 12). For comparison, we present there (Col. 13) also the average optical depths of line formation based on the contribution function to the emergent total (line + continuum) radiation, $\log \tau_{5000}^{l+c}$. It was emphasized by Achmad et al. (1991) that the first approach provides a more realistic depth of line formation, in particular, for weak lines, for which the continuum contribution shifts an average depth formation inward inside the atmosphere. This effect becomes far more important in the case of a stratified atmosphere. When the contribution function to the total radiation is used, the formation depth of most Pr II lines is shifted well below the enhanced Pr abundance layer. The nonLTE formation depths for the $\mathrm{H} \alpha$ core were computed according to Mashonkina et al. (2008). It can be seen from Fig. 11 that, with the stratified Pr and Nd abundance distributions found based on non-LTE line formation, we derive a consistent picture that is typical of running pulsation wave where the amplitudes and phases increase towards upper layers. A gap of $\sim 0.5$ dex at $\log \tau_{5000} \sim-4.5$ may be caused by the uncertainties in the modelling.

\subsection{The uncertainties in Pr stratification analysis}

How do the uncertainties in atomic parameters and ignoring the magnetic intensification of spectral lines affect our final results?

Photoionization cross-sections. The statistical equilibrium of Pr II/III in the stratified atmosphere is defined mainly by enhanced photoionization of Pr II. We perform test calculations using various photoionization cross-sections. When the hydrogenous photoionization cross-sections are increased by a factor of 30 , the overionization of Pr II is amplified and $\Delta_{\text {NLTE }}$ increases by 0.03 dex to 0.05 dex for different Pr II lines. The uncertainty in photoionization cross-sections is far less important for Pr III. Reducing the hydrogenous photoionization cross-sections by a factor of 100 has a much larger effect on both the Pr II and Pr III lines. In this case, the layer of enhanced Pr must be shifted outward by $\Delta \log \tau_{5000} \simeq 0.5$ to ensure that the abundances of Pr determined for two ionization stages remain consistent. The obtained Pr abundance distribution is shown by a dashed curve in the top panel of Fig. 7. We emphasize that even with the lowest photoionization cross-sections the ionization equilibrium Pr II/Pr III deviates significantly from that of TE, and non-LTE removes approximately $1.5 \mathrm{dex}$ of the difference between the Pr abundances derived from the Pr II and Pr III lines under the LTE assumption. As discussed in our earlier paper (Paper I), the adopted hydrogenic approximation provides, probably, a lower limit to the photoionization cross-sections for the levels in the rare-earth elements. So, we under- rather than overestimate non-LTE effects for Pr II/III in our calculations.

Collision rates. In SE computations, we assume an effective collision strength equal to $\Upsilon=1$ for every forbidden transition, which may be incorrect. The $R$-matrix method calculations for electron impact excitation in Ca II (Meléndez et al. 2007) indicate that $\Upsilon$ depends on the transition energy separation. For example, at the electron temperatures with which we are concerned, $\Upsilon>30$ for the forbidden transitions with $\Delta E_{i j}<0.1 \mathrm{eV}$, while $\Upsilon \leq 0.1$ if $\Delta E_{i j}>10 \mathrm{eV}$. For the fine-structure transitions in Fe II, the $R$-matrix method predictions of Ramsbottom et al. (2007) imply that $\Upsilon \geq 5$. We perform non-LTE calculations for Pr II/III assuming that for the forbidden transitions, $\Upsilon$ depends on the transition energy separation as follows: $\log \Upsilon=1.5$ for $\Delta E_{i j}<0.1 \mathrm{eV}, \log \Upsilon=-0.38 \Delta E_{i j}+1.54$ for $\Delta E_{i j}=0.1 \mathrm{eV}-$ $4 \mathrm{eV}, \log \Upsilon=-0.17 \Delta E_{i j}+0.67$ for $\Delta E_{i j}=4 \mathrm{eV}-10 \mathrm{eV}$, and $\log \Upsilon=-1$ for $\Delta E_{i j} \geq 10 \mathrm{eV}$. These approximations are based on the extensive data set of Meléndez et al. (2007) for forbidden transitions in Ca II. With stronger collisional coupling of 

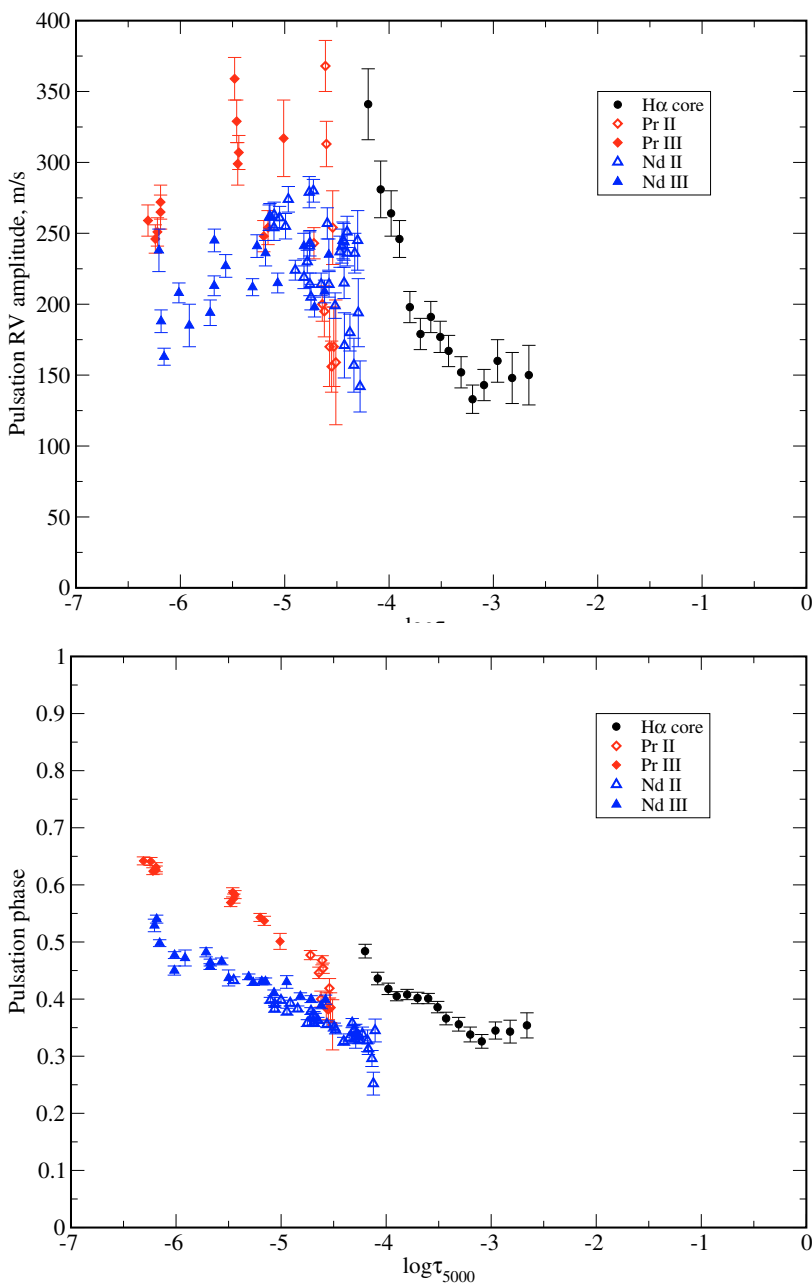

Fig. 11. Variations in pulsation radial-velocity amplitude (top panel) and phase (bottom panel) for the lines of Pr II (open diamonds), Pr III (filled diamonds), Nd II (open triangles), Nd III (filled triangles), and the $\mathrm{H} \alpha$ core (filled circles) as a function of the optical depth in the atmosphere of the roAp star HD 24712 with the stratified distribution of Pr and $\mathrm{Nd}$.

the low-excitation levels to the PrII ground state, the departures from LTE are weakened for the Pr II transitions arising from the levels with $E_{\text {exc }}<0.8 \mathrm{eV}$, and they are strengthened for the remaining transitions in Pr II and all transitions in Pr III. The Pr abundance distribution obtained from these test calculations is shown by a dotted curve in the top panel of Fig. 7. We find that no revision is required outside $\log \tau_{5000}=-6.5$ and inside $\log \tau_{5000}=-4$ compared to the distribution found in Sect. 6.2, while the praseodymium abundance is decreased by up to 0.2 dex in-between.

Magnetic field. The magnetic field effects are taken into account in this study approximately by introducing a pseudomicroturbulence. First, this works only for strong spectral lines. Second, magnetic field and microturbulence affect the saturated line profile in different ways. A magnetic field splits the line into its Zeeman components making the line less deep (magnetic desaturation) and broader, while microturbulence does not affect the line depth, but increases the half-width of the line profile. As shown in Sect. 6.1, accounting for Zeeman splitting results in small changes of $0.16 \mathrm{dex}$, on average, in the derived Pr abundances compared with those determined without a magnetic field. This effect cannot influence our conclusion about the stratified distribution of $\operatorname{Pr}$ in the atmosphere of HD 24712.
Zeeman splitting may also result in line formation depths that differ from those for turbulently broadened lines and, therefore, in a different location of the Pr enriched layer. We estimate this effect using the strongest Pr III $\lambda 5300 \AA$ line. Zeeman splitting of this line is represented by the three components. By comparison of the computed single line with $V_{\text {mic }}=1.05 \mathrm{~km} \mathrm{~s}^{-1}$ and the triplet with zero microturbulence, we deduce that the magnetic desaturation may produce only a 0.2 dex decrease in the Pr abundance of the layer, and shift the Pr abundance profile downward, by 0.35 dex in a $\log \tau_{5000}$ scale.

Thus, the uncertainty in the used atomic data about photoionization cross-sections and electron impact excitation cross-sections and our neglect of the magnetic field in nonLTE analysis cannot change our conclusion about the existence of an inhomogeneous distribution of Pr in the atmosphere of HD 24712.

\section{Conclusions}

We have presented a comprehensive model atom for Pr II/III based on both measured and calculated energy levels. NonLTE line formation for an extended list of Pr II and Pr III lines was considered for both the Sun and the temperatures characteristic of A type stars, $T_{\text {eff }}=7250 \mathrm{~K}-9500 \mathrm{~K}$. At $T_{\text {eff }} \leq 8000 \mathrm{~K}$, Pr II represents the state in which the majority of the element exists and departures from LTE for the Pr II lines are small. They are caused mainly by a deviation in the line source function from the Planck function and may result in non-LTE abundance corrections of different sign for various lines. Overionization of Pr II at $T_{\text {eff }} \geq 8500 \mathrm{~K}$ leads to depleted total absorption in the Pr II lines and positive $\Delta_{\text {NLTE }}$ that grows with temperature. NonLTE leads to strengthening of the Pr III lines and negative abundance corrections over the full range of stellar parameters. As Pr III becomes the majority species, $\Delta_{\text {NLTE }}$ of the Pr III lines decreases in absolute value.

Using the modern laboratory data for oscillator strengths of the Pr II lines, improved partition function of Pr II based on the laboratory and the calculated energy levels, we determine the non-LTE abundance of praseodymium in the solar atmosphere, $\log (\operatorname{Pr} / \mathrm{H})_{\odot}=-11.15 \pm 0.08$ and $\log (\mathrm{Pr} / \mathrm{H})_{\odot}=-11.10 \pm 0.08$, for the ATLAS9 and the Holweger \& Müller (1974) models, respectively. The Pr abundance based on the theoretical model is 0.05 dex to 0.10 dex higher than the meteoritic value recommended by Grevesse et al. (1996), Palme \& Jones (2005), Lodders (2003), or Asplund et al. (2005), but the deviation corresponds to an uncertainty that is still within the calculated error bars.

For the roAp star HD 24712, the element abundances from two ionization stages, Pr II and Pr III, reveal a discrepancy of two orders of magnitude if the atmosphere is assumed to be chemically homogeneous. Neither non-LTE, nor magnetic effects can be responsible for such an abundance anomaly.

Introducing the layer with a strongly enhanced Pr abundance in the outer atmosphere provide a natural possibility of describing the lines of both ionization stages, Pr II and Pr III, for the single element distribution. We found that the required Pr overabundance in the layer was $[\mathrm{Pr} / \mathrm{H}] \geq 4$ at $\log \tau_{5000}<-4$. The inferred praseodymium and neodymium stratifications provided a possibility of explaining the distributions of the pulsational characteristics (radial-velocity amplitudes and phases) in the significant part of the atmosphere of HD 24712, where the lines of these elements as well as the core of the $\mathrm{H} \alpha$ line are formed.

Similar abundance profiles found empirically for $\mathrm{Nd}$ (Paper I) and Pr in the atmosphere of HD 24712 point to some 
common physical mechanisms producing accumulation of these two elements in the uppermost atmospheric layers. The main mechanism can be radiatively driven diffusion. The theoretical diffusion calculations of LeBlanc \& Monin (2004) for $\mathrm{Cr}$ and $\mathrm{Fe}$ and of Alecian \& Stift (2007) for Mg, Si, Ca, Ti, and Fe predict the existence of abundance jumps of several orders of magnitude in stable stellar atmospheres with effective temperatures that are characteristic of Ap stars. Similar abundance profiles are derived empirically for these elements in the atmospheres of roAp stars (Ryabchikova 2008). In contrast to REEs, Mg to Fe are accumulated in the lower atmosphere. Due to difficulties with the atomic data, no diffusion calculations were performed for the REEs to be able to confirm the validity of the empirical distributions, although the situation is improving and we hope to see the first REE diffusion calculations in the future.

We are planning to extend our non-LTE line formation study to other REE that exhibit a discrepancy between the element abundances derived from lines of two different ionization stages in roAp stars. As shown for $\mathrm{Nd}$ in Paper I and for Pr in the present work, the departures from LTE for the lines of the first and the second ions are of the opposite sign, and they are significant if the element is concentrated in the uppermost atmospheric layers where collisions are inefficient in establishing thermodynamic equilibrium. In such a case, a stratification analysis for REE should be performed based on non-LTE line formation.

Acknowledgements. The authors are grateful to J.-F. Wyart for help in calculations of PrII and Pr III spectra. This research was supported by the Russian Foundation for Basic Research with grant 08-02-00469-a, by the Presidium RAS Programme "Origin and evolution of stars and galaxies", and by the Leading Scientific School grants 4224.2008.2 and 4354.2008.2. TR also acknowledges a partial support from the Austrian Science Fund (FWF-P17580N2).

\section{References}

Achmad, I., de Jager, C., \& Nieuwenhuijzen, H. 1991, A\&A, 250, 445 Alecian, G., \& Stift, M. J. 2007, A\&A, 475, 659

Allard, N. F., Drira, I., Gerbaldi, M., et al. 1998, A\&A, 335, 1124

Anders, E., \& Grevesse, N. 1989, Geochim. Cosmochim. Acta, 53, 197

Anstee, S. D., \& O'Mara, B. J. 1995, MNRAS, 276, 859

Asplund, M., Grevesse, N., \& Sauval, A. J. 2005, ASP Conf. Ser., 336, 25

Bard, A., Kock, A., \& Kock, M. 1991, A\&A, 248, 315

Biémont, E., Grevesse, N., \& Hauge, Ø. 1979, Sol. Phys., 61, 17

Biémont, E., Garnir, H. P., Palmeri, P., et al. 2001, Phys. Rev. A, 64, 2503

Biémont, E., Lefebvre, P. H., Quinet, P., et al. 2003, EPJ D, 27, 33

Brewer, L. 1971, JOSA, 61, 1666

Butler, K., \& Giddings, J. 1985, Newsletter on the analysis of astronomical spectra 9, University of London

Canuto, V. M., Goldman, I., \& Mazzitelli, I. 1996, ApJ, 473, 550

Castelli, F., \& Kurucz, R. 2001, A\&A, 372, 260

Cowan, R. D. 1981, The Theory of Atomic Structure and Spectra, Univ. of (Berkeley California USA: California Press)

Cowley, C. R., \& Bord, D. J. 1998, in The scientific impact of the Goddard High Resolution Spectrograph, ASP Conf. Ser., 143, 346

Cowley, C.R., Ryabchikova, T., Kupka, F., et al. 2000, MNRAS, 317, 299

Drawin, H.-W. 1961, Z. Phys., 164, 513

Fuhrmann, K., Pfeiffer, M., Frank, C., et al. 1997, A\&A, 323, 909

Furman, B., Stefanska, D., Stachowska, E., et al. 2001, EPJ D, 17, 275

Gelbmann, M., Ryabchikova, T. A., Weiss, W. W., et al. 2000, A\&A, 356, 200

Ginibre, A. 1989a, Phys. Scr., 39, 694
Ginibre, A. 1989b, Phys. Scr., 39, 710

Ginibre, A. 1990, Atomic Data and Nuclear Data Tables, 44, 1

Grevesse, N., Noels, A., \& Sauval, A. J. 1996, ASP Conf. Ser., 99, 117

Grupp, F. 2004, A\&A, 420, 289

Heiter, U., Kupka, F., van 't Veer-Menneret, C., et al. 2002, A\&A, 392, 619

Holweger, H., \& Müller, E. A. 1974, Sol. Phys., 39, 19

Ivarsson, S., Litzén, U., \& Wahlgren, G. 2001, Phys. Scr., 64, 455

Ivarsson, S., Wahlgren, G., \& Ludwig, H.-G. 2003, BAAS, 35, 1421

Kato, K. 2003, PASJ, 55, 1133

Kochukhov, O. 2003, A\&A, 404, 669

Kochukhov, O. 2007, in Physics of Magnetic Stars, ed. I. I. Romanyuk, D. O.

Kudryavtsev, Nizhnij Arkhyz, 109

Kochukhov, O., Khan, S., \& Shulyak, D. 2005, A\&A, 433, 671

Kupka, F., Piskunov, N., Ryabchikova, T. A., et al. 1999, A\&AS, 138, 119

Kurucz, R. L. 1994a, Opacities for Stellar Atmospheres, CD-ROM (Cambridge, Mass), 2

Kurucz, R. L. 1994b, SYNTHE Spectrum Synthesis Programs and Line Data, CD-ROM (Cambridge, Mass), 18

Kurucz, R. L., \& Bell, B. 1995, Atomic Line Data. Kurucz CD-ROM (Cambridge, Mass), 23

Kurucz, R. L., Furenlid, I., Brault, J., \& Testerman, L. 1984, NSO Atlas No. 1, Solar Flux Atlas from 296 to $1300 \mathrm{~nm}$, Sunspot, NSO

Lage, C. S., \& Whaling, W. 1976, JQSRT, 16, 537

LeBlanc, F., \& Monin, D. 2004, The A-Star Puzzle, ed. J. Zverko, W. W. Weiss, J. Žižňovský, \& S. J. Adelman, IAUS, 224, 193

Li, R., Chatelain, R., Holt, R. A., et al. 2007, Phys. Scr., 76, 577

Lodders, K. 2003, ApJ, 591, 1220

Martin, W. C., Zalubas, R., \& Hagan, L. 1978, Atomic energy levels - The Rare Earth Elements, NSRDS-NBS 60, U.S. Gov. Print. Off., Washington

Mashonkina, L. 2008, in Non-LTE line formation for trace elements in stellar atmospheres, ed. R. Monier, B. Smalley, Ph. Stee, \& G. Wahlgren, EAS Publ. Ser., in press

Mashonkina, L., Ryabchikova, T. A., \& Ryabtsev, A. N. 2005, A\&A, 441, 309 (Paper I)

Mashonkina, L., Zhao, G., Gehren, T., et al. 2008, A\&A, 478, 529

Meggers, W. F, Corliss, C. H., \& Scribner, B. F. 1975, NBS Monograph 145, U.S. Gov. Print. Off., Washington, D.C.

Meléndez, M., Bautista, M. A., \& Badnell, N. R. 2007, A\&A, 469, 1203

Palme, H., \& Jones, A. 2005, in Meteorites, Comets and Planets: treatise on Geochemistry, 1, ed. A. M. Davis, Elsevier Publ., Amsterdam, The Netherlands, 41

Palmeri, P., Quinet, P., Fremat, Y., et al. 2000, ApJS, 129, 367

Ramsbottom, C. A., Hudson, C. E., Norrington, P. H., \& Scott, M. P. 2007, A\&A, 475,765

Reetz, J. K. 1991, Diploma Thesis, Universität München

Ryabchikova, T. 2008, Contr. Astron. Obs. Skalnaté Pleso, 38, 257

Ryabchikova, T., Piskunov, N., Kochukhov, O., et al. 2002, A\&A, 384, 545

Ryabchikova, T., Nesvacil, N., Weiss, W. W., et al. 2004, A\&A, 423, 705

Ryabchikova, T., Ryabtsev, A., Kochukhov, O., \& Bagnulo, S. 2006, A\&A, 456, 329

Ryabchikova, T., Sachkov, M., Kochukhov, O., \& Lyashko, D. 2007a, A\&A, 473, 907

Ryabchikova, T., Sachkov, M., Weiss, W. W., et al. 2007b, A\&A, 462, 1103

Ryabchikova, T. A., Landstreet, J. D., Gelbmann, M. J., et al. 1997, A\&A, 327, 1137

Ryabchikova, T. A., Savanov, I. S., Malanushenko, V. P., \& Kudryavtsev, D. O. 2001, Astron. Rep., 45, 382

Ryabchikova, T. A., Kochukhov, O., \& Bagnulo, S. 2008, A\&A, 480, 811

Rybicki, G. B., \& Hummer, D. G. 1991, A\&A, 245, 171

Rybicki, G. B., \& Hummer, D. G. 1992, A\&A, 262, 209

Scholl, T., Holt, R., Masterman, D., et al. 2002, Can. J. Phys., 80, 713

Smith, W. W., \& Gallagher, A. 1966, Phys. Rev., 145, 26

Steenbock, W., \& Holweger, H. 1984, A\&A, 130, 319

Takeda, Y. 1994, PASJ, 46, 53

van Regemorter, H. 1962, ApJ, 136, 906

Wickliffe, M. E., Lawler, J. E., \& Nave, G. 2000, JQSRT, 66, 363 